Journal for ImmunoTherapy of Cancer

\title{
THADA drives Golgi residency and upregulation of PD-L1 in cancer cells and provides promising target for immunotherapy
}

To cite: Li C, Chi H, Deng S, et al. THADA drives Golgi residency and upregulation of PD-L1 in cancer cells and provides promising target for immunotherapy. Journal for ImmunoTherapy of Cancer 2021;9:e002443. doi:10.1136/ jitc-2021-002443

- Additional supplemental material is published online only. To view, please visit the journal online (http://dx.doi.org/10. 1136/jitc-2021-002443).

Accepted 05 July 2021
Check for updates

\section{(c) Author(s) (or thei} employer(s)) 2021. Re-use permitted under CC BY-NC. No commercial re-use. See rights and permissions. Published by BMJ.

For numbered affiliations see end of article.

\section{Correspondence to}

Dr Jie Xu; jie_xu@fudan.edu.cn

\section{ABSTRACT}

Background The abnormal upregulation of programmed death-ligand 1 (PD-L1) in cancer cells inhibits T cellmediated cytotoxicity, but the molecular mechanisms that drive and maintain PD-L1 expression are still incompletely understood.

Methods Combined analyses of genomes and proteomics were applied to find potential regulators of PD-L1. In vitro experiments were performed to investigate the regulatory mechanism of $\mathrm{PD}-\mathrm{L} 1$ by thyroid adenoma associated gene (THADA) using human colorectal cancer (CRC) cells. The prevalence of THADA was analyzed using CRC tissue microarrays by immunohistochemistry. T cell killing assay, programmed cell death 1 binding assay and MC38 transplanted tumor models in C57BL/6 mice were developed to investigate the antitumor effect of THADA. Results THADA is critically required for the Golgi residency of $\mathrm{PD}-\mathrm{L} 1$, and this non-redundant, coat protein complex II (COPII)-associated mechanism maintains PD-L1 expression in tumor cells. THADA mediated the interaction between PD-L1 as a cargo protein with SEC24A, a module on the COPII trafficking vesicle. Silencing THADA caused absence and endoplasmic reticulum (ER) retention of PDL1 but not major histocompatibility complex-I, inducing PD-L1 clearance through ER-associated degradation. Targeting THADA substantially enhanced T cell-mediated cytotoxicity, and increased CD8+ T cells infiltration in mouse tumor tissues. Analysis on clinical tissue samples supported a potential role of THADA in upregulating PD-L1 expression in cancer.

Conclusions Our data reveal a crucial cellular process for PD-L1 maturation and maintenance in tumor cells, and highlight THADA as a promising target for overcoming PDL1-dependent immune evasion.

\section{BACKGROUND}

Accumulating evidence reveals that immune checkpoint blockade (ICB) has reshaped the treatment landscape of many malignancies. ${ }^{1}$ Among all ICB therapies, blockade of programmed cell death 1 (PD-1) and its ligand programmed death-ligand 1 (PD-L1) with monoclonal antibodies has demonstrated remarkable clinical benefit in various solid tumors. ${ }^{2-4}$ However, considerable challenges, such as lack of durable remission, low response rate, drug resistance and immunerelated adverse effects, still remain to be addressed..$^{5-10}$ It is generally considered that PD-L1 expressed on the tumor cell surface is upregulated in the tumor microenvironment to activate PD-1 on CD8 T cells, delivering the co-inhibitory signals and thereby suppressing $\mathrm{T}$ cell function. ${ }^{11}$ However, except being presented on the tumor cell surface, PD-L1 is also dynamically circulating inside tumor cells $^{1213}$ as well as excreted into extracellular space via the exosomes, ${ }^{14}{ }^{15}$ contributing to the resistance to anti-PD-L1 antibodies. Therefore, further exploration of the track of PD-L1 is urgently needed to maximize the therapeutic efficiency of PD-L1 blockade.

As a type I transmembrane glycoprotein, PD-L1 undergoes intricate secretory pathway throughout the lifetime. Generally, the nascent peptide will co-translationally translocate to the endoplasmic reticulum (ER) to initiate its early secretory pathway. After entering the ER lumen, PD-L1 is extensively $\mathrm{N}$-glycosylated with the help of multiple ER chaperones and enzymes while aberrant glycosylation leads to the degradation of PD-L1. ${ }^{16} 17$ GSK3 $\beta$ phosphorates the nonglycosylated PD-L1 to induce the proteasomemediated degradation of PD-L1 by $\beta$-TrCP. ${ }^{16}$ Numerous compounds, involving metformin, resveratrol and BMS1166, are all demonstrated to disrupt normal glycosylation of PD-L1 in different manners. ${ }^{18-20}$ In addition, some ER chaperones, such as GRP78 and Sigma1, are also indicated to interact with and stabilize PD-L1. ${ }^{21} 22$

After a series of modifications in the ER and Golgi apparatus, PD-L1 protein gets matured and continues its vigorous intracellular transport. CMTM6 is proposed to 
specifically bind PD-L1 to induce the continuous redistribution of PD-L1 between the recycling endosome (RE) and the cell membrane, and thus help PD-L1 evade lysosomal degradation. ${ }^{12}$ Our recent studies have also deeply elucidated the transport mechanisms of PD-L1. Palmitoylation of PD-L1 effectively maintains its expression both on the cell membrane and the RE by escaping from endosomal sorting complex required for transport (ESCRT) sorting to the multivesicular bodies (MVB) and then lysosomal degradation. ${ }^{23}$ Amlodipine markedly provokes selective autophagy of PD-L1 highly enriched on the RE. ${ }^{24}$ In addition, HIP1R can directly bind PD-L1 and deliver it to lysosomes for degradation. ${ }^{25}$ Of note, emerging studies have revealed that tumor cells can express PD-L1 on the cell membrane and release exosomes with PD-L1 on the surface via the MVBs, which remarkably promotes tumor progression and immune evasion. ${ }^{14} 15$ The ESCRT-associated protein ALIX depletion conducts the redistribution of PD-L1, reducing exosomal PD-L1 but increasing PD-L1 on the tumor cell surface.$^{26}$ Together, substantial evidence has constructed the sophisticated PD-L1 trafficking machineries involving the protein maturation in the ER as well as internalization, recycling and degradation inside the cell and secretion outside the cell. However, as the most vital part of the early secretory pathway, it remains poorly documented how tons of PD-L1 proteins that complete processing in the ER transfer to the Golgi apparatus.

In eukaryotic cells, protein trafficking from the ER to the Golgi complex is critically dependent on the coat protein complex II (COPII) vesicles. ${ }^{27}$ There are five highly conserved components to form the basic machinery of COPII coat, the small GTPase Sar1, Sec23, Sec24, Sec13 and Sec31. Cargo proteins with correct structure and modification that checked by ER quality control system (ERQC) exit from the ER at a distinct region termed the ER exit sites where COPII coat modules are enriched. The activated Sar1-GTP inserts into the ER membrane and initiates the assembly of COPII coat by activating and binding to Sec23 and thus recruiting the heterodimeric Sec23/24 as the inner coat and Sec13/ Sec31 complex as the outer coat in sequence. ${ }^{27-29}$ Especially, Sec24 serves as the sole adaptor that sorts and captures specific cargo proteins by direct interaction with the substrates. Studies have identified four isoforms of Sec24 in human cells, termed Sec24A-D, respectively, with diverse non-overlapping interfaces, which largely extend the selectivity of cargo proteins accommodated by COPII vesicles. ${ }^{30-34}$ Approximately one-third of the proteins must go through the COPII vesicle trafficking, however, only a limited number of peptide sequences and conformational epitopes have been related to cargo sorting. How different isoforms of Sec24 precisely orchestrate the specific transportation of a large variety of cargoes remains poorly characterized.

Remarkably, there are some proteins identified as cargo receptors assisting in the COPII vesicle transport of particular transmembrane proteins. Studies indicate that the cornichon protein family and its yeast homologues Erv14 are required for coupling a series of polytopic transmembrane cargoes to Sec24. ${ }^{35-40}$ For instance, CNIH4, the human cornichon homologue 4 , is suggested as a selective intermediate to interacts with $G$ protein-coupled receptors (GPCRs) as well as Sec23 and two subunits of Sec24 (Sec24B and Sec24D), thereby modulating the ER-to-Golgi trafficking of GPCRs. Abnormal expression of CNIH4 leads to the retention and subsequent proteasomal degradation of GPCRs. ${ }^{40}$ In addition, SCAP binds to SREBPs to mediate its COPII vesicle trafficking depending on different concentration of cholesterol in the ER. ${ }^{41}{ }^{42}$ Hence, it is hypothesized that some particular cargo proteins require a dual interaction with both Sec24 and the cargo receptor for COPII vesicle delivery, whereas only a few cargo receptors have been characterized up to now.

Generally, ERQC approves the egress of correctly processed proteins via COPII vesicles while sequesters aberrant proteins in the ER lumen and subsequently degrades the proteins via ER-associated degradation (ERAD).$^{43} 44$ The HRD1 complex residing on the ER membrane is proposed as the major ERAD operator. The key subunits of HRD1 complex containing the lectinlike proteins OS-9 and XTP3-B, as well as E3 ubiquitin ligase HRD1, synergistically manipulate the recognition, targeting and retrotranslocation of the ERAD substrates to ferry the substrate proteins into the cytoplasm for the ubiquitin-proteasome degradation. ${ }^{45}$

In the present study, we characterize thyroid adenoma associated gene (THADA) as an indispensable regulator of PD-L1 maturation, participating in the PD-L1-specific ER export to Golgi by coupling PD-L1 to Sec24A-mediated COPII vesicles, thereby stabilizing the Golgi residency of PD-L1 and maintaining PD-L1 expression in tumor cells. THADA highly expressed in human colorectal cancer (CRC) tissue samples is significantly positive correlated with PD-L1 expression, and depletion of THADA selectively contributes to ER retention and ERAD degradation of PD-L1 without compromising major histocompatibility complex (MHC)-I expression, remarkably promoting $\mathrm{T}$ cell cytotoxicity and antitumor effect in vitro and in vivo. Our work provides new insight into PD-L1 trafficking machineries in the early secretory pathway, highlighting THADA as an alternative target for PD-L1-dependent tumor immunotherapy.

\section{MATERIALS AND METHODS \\ Tumor models}

C57BL/6 mice (female, 6 weeks old, purchased from Shanghai Lingchang Biotechnology) were acclimated for 1 week and then randomized into three groups $(n=8$ for each group, 24 mice in total), and injected with $1 \times 10^{6}$ mouse colorectal MC38 stable clones subcutaneously in the flank (control short hairpin RNA (shRNA) lentivirus, THADA shRNA or THADA shRNA+PD-L1 OE, the establishment of MC38 stable clones as described 
later). The tumor sizes were recorded every 2-3 days using a vernier caliper and calculated with the formula $1 / 2 \times{\mathrm{A} \times \mathrm{a}^{2}}^{2}$ (A and a, respectively, denote the length and the width of the tumor). For anti-PD-L1 treatment, mice inoculated with MC38 stable clones (THADA shRNA or control shRNA lentivirus) were randomized to receive anti-PD-L1 antibody (Bioxcell, clone 10F.9G2, BE0101, 5 $\mathrm{mg} / \mathrm{kg}$, intraperitoneal, once a week) or isotype-matched control antibody (Bioxcell, BE0090) ( $\mathrm{n}=8$ for each group, 32 mice in total). In accordance with the ethical guidelines, mice were sacrificed once the tumor volume reached $1500 \mathrm{~mm}^{3}$ or ulcers happened. All the mice were sacrificed on day 19 after inoculation and the tumors were resected and weighed, and pretreated for further experiments. The number of mice assigned to each treatment was selected to provide sufficient statistical power to discern significant differences in tumor control and overall survival, based on prior experience with the model. The experiments were performed in Fudan University and Innomodels Biotechnology (Beijing), China.

\section{Cell culture}

All cell lines including human CRC RKO, LoVo, HCT116 cells, human breast cancer MDA-MB-231 cells, melanoma A375 cells were purchased from American Type Culture Collection and mouse CRC MC38 cells were purchased from Kerafast and all mycoplasma free. RKO and LoVo cells were incubated in RPMI-1640 (Gibco) with 10\% fetal bovine serum (FBS) (Gibco). HCT116 cells were incubated in McCoy'5A (Gibco) with 10\% FBS (Gibco). MDA-MB-231 cells, A375 and MC38 cells were incubated in Dulbecco's Modified Eagle Medium (DMEM) (Gibco) with $10 \%$ FBS (Gibco). All cells were cultured at $37^{\circ} \mathrm{C}$ under $5 \% \mathrm{CO}_{2}$.

\section{Antibodies and reagents}

The primary antibodies for PD-L1 (13684 and 29122, CST), PD-L1 (17952-1-AP, Proteintech), PD-L1 (329708, BioLegend), GAPDH (KC-5G5, KANGCHEN), MHC-I (ab70328, Abcam), MHC-I (15240-1-AP, Proteintech), THADA (ab122260, Abcam), THADA (12909-1-AP, Proteintech), Sec24A (ab102660, Abcam), Sec24A (15958-1-AP, Proteintech), Flag-tag (2368, CST), HA-tag (3724, CST), Myc-tag (2276, CST), XTP3-B/ERLEC1 (ab181166, Abcam), OS-9 (ab109510, Abcam), HRD1/ SYVN1 (13473-1-AP, Proteintech), 58K (ab27043, Abcam), GRP94 (ab3674, Abcam), CD8a (D4W2Z; CST), CD8a (CL488-65069; Proteintech), ubiquitin (3936, CST), granzyme B (17215, CST), sodium potassium ATPase (ab76020, Abcam) were all commercially available. Reagents involving recombinant human interferon (IFN) $-\gamma$ protein (285-IF, R\&D Systems), rhPD-1/ FC chimera protein (1086-PD-050, R\&D Systems), recombinant human IL-2 (589102, BioLegend), cycloheximide (C1988, Sigma-Aldrich), MG132 (M7449, Sigma-Aldrich), chloroquine (CQ) (C6628, SigmaAldrich), eeyarestatin I (Eer I) (10012609, Cayman) and 4',6-diamidino-2-phenylindole (DAPI) (0100-20;
SouthernBiotech) were also purchased from the indicated suppliers.

\section{Plasmids construction}

The pcDNA3.1-Flag-PD-L1 vector was purchased from Generay Biotech (Shanghai), established by inserting synthesized complementary DNA (cDNA) encoding $3 \times$ Flag tag and PD-L1 (clone R8443-2) into pcDNA3.1 vector using EcoRI/XhoI MCS. The pcDNA3.1-HA-Sec24A was purchased from Generay (Shanghai), constructed by inserting synthesized cDNA encoding HA tag and Sec24A (human) into the pcDNA3.1 vector, using the EcoRI/ XhoI multiple cloning sites (MCS). The pcDNA3.1-MycTHADA was purchased from Genechem (Shanghai), generated by inserting synthesized cDNA encoding Myc tag and THADA fragment (human) into the pcDNA3.1 vector, using the EcoRI/XhoI MCS. THADA-His vector was purchased from General Biosystem (Anhui), generated by inserting synthesized cDNA encoding THADA fragment (human) into C-his-tag, using BamHI/Notl site. All vectors were checked by sequencing and western blot analysis with specific antibodies in which the observed molecular weights were in concordance with the predicted molecular weights.

\section{Transfection of plasmids or small interfering RNAs}

Tumor cells were seeded in 6-well plates to reach a density of $\sim 40 \%$ at the time of transfection. Twenty-fourhours later, transfection was performed using 1.5-2 $\mu \mathrm{g}$ plasmid together with 4.5-6 $\mu \mathrm{L}$ FuGENE HD (Promega) and $100 \mu \mathrm{L}$ Opti-MEM for per well according to the manufacturer's guidance. The negative control in each experiment was cells mock-transfected with empty control vectors. All the small interfering RNAs (siRNAs) were purchased from Genepharma for the depletion of THADA, Sec24A, OS-9, XTP3-B and HRD1, using DharmaFECT 1 transfection reagent (Invitrogen) according to the manufacturer's directions. There was also a non-specific siRNA as a negative control for each experiment. The sequences of siRNAs are summarized in online supplemental table 1.

\section{Establishment of PD-L1 stable cells}

We chose human CRC HCT116 cells to establish PD-L1 stable cell strains for its pretty low endogenous PD-L1 expression. First, we transfected ectopic FLAG-tagged PD-L1 into HCT116 cells following the procedures described above. We also set a blank vector control and an empty control, respectively. After approximately 2-week incubation in McCoy's 5A (Gibco) supplemented with $800 \mu \mathrm{g} / \mathrm{mL}$ G418 (Gibco BRL) with refreshing the medium every 2-3 days, the single colonies were picked and verified by immunoblots. Given the results of ectopic PD-L1 expression level determined by western blot analysis, we chose a colon with highest PD-L1 expression and generated the HCT116 ectopic PD-L1 stable cells.

\section{Lentivirus transduction}

All the lentivirus for THADA silencing were purchased from Genepharma. The sequences of lentiviral shRNA are 
summarized in online supplemental table 2. Mouse CRC MC38 cells were seeded in a 6-well plate and infected with LV3-THADA-GFP lentiviral shRNA, LV3-THADA-GFP lentiviral shRNA and LV-Cd274-EGFP, or the blank vector in DMEM complete medium supplemented with $5 \mu \mathrm{g} /$ $\mathrm{mL}$ polybrene. After 24 hours incubation, the medium was refreshed with DMEM complete medium containing $5 \mu \mathrm{g} / \mathrm{mL}$ puromycin. The medium was refreshed every 2-3 days for 2 weeks. The transfection efficiency and the expression of THADA and PD-L1 were confirmed using fluorescent microscopy, qRT-PCR and immunoblots before use.

\section{Quantitative real-time PCR assays}

The cells cultured in the 6-well plate were harvested and lysed with $1 \mathrm{~mL}$ Trizol reagent (Invitrogen) per well. The RNA was reverse transcribed using the PrimeScript RT Reagent Kit (Takara) following the manufacturer's instructions. Quantitative real-time PCR was performed using an Applied Biosystem 7900HT Sequence Detection System (Applied Biosystems) with GoTaq RT-qPCR system (Promega). The $\mathrm{Ct}$ values were normalized by $2-\Delta \Delta \mathrm{Ct}$ and presented as relative fold change. GAPDH and $\beta$-actin were applied as the reference genes. DNA primer sequences are supplied in online supplemental table 3 .

\section{Immunoblotting and co-immunoprecipitation}

Cells were lysed with RIPA buffer (Beyotime) supplemented with $1 \%$ cocktail containing proteinase and phosphatase inhibitors and phenylmethylsulfonyl fluoride (PMSF) (KC-440, KANGCHEN) and collected to fresh $1.5 \mathrm{~mL}$ microfuge tubes. The cell lysates were centrifuged at $12,000 \mathrm{rpm}$ for $15 \mathrm{~min}\left(4^{\circ} \mathrm{C}\right)$ and supernatant was transferred to fresh $1.5 \mathrm{~mL}$ microfuge tubes. The protein concentration was determined by BCA Protein Assay Kit (ThermoFisher Scientific); $5 \times$ sodium dodecyl sulfatepolyacrylamide gel electrophoresis (SDS-PAGE) loading buffer (Beyotime) was diluted to $1 \times$ with protein sample, heating at $95^{\circ} \mathrm{C}$ for $8-10 \mathrm{~min}$. The protein extracts were subjected to appropriate concentrations of SDS-PAGE for electrophoresis and transferred to homopolymers and copolymers(PVDF) membranes (Bio-Rad). Membranes were blocked with 5\% bovine serum albumin (0332100G, Amresco) for 1 hour at room temperature, and then incubated with primary antibodies overnight at $4^{\circ} \mathrm{C}$. Membranes were then incubated with secondary HRP-conjugated antibodies (KANGCHEN) at room temperature for 1 hour. Before and after the incubation, membranes were washed five times with $1 \times$ Tris-buffered saline, $0.1 \%$ Tween 20 (TBST), respectively. Ultimately, membranes were examined by ChemiDoc imaging system (Bio-Rad). For co-immunoprecipitation (Co-IP), cells were harvested and lysed with IP Lysis Buffer (ThermoFisher Scientific) supplemented with $1 \%$ cocktail of proteinase and phosphatase inhibitor and PMSF (ThermoFisher Scientific). The cell lysates were centrifuged at $12,000 \mathrm{rpm}$ for $2 \mathrm{~min}$. After DNase treatment for $20 \mathrm{~min}$ at room temperature, $16 \mu \mathrm{L}$ of each sample $(300 \mu \mathrm{L})$ supplemented with $4 \mu \mathrm{L} 5 \times$ SDS-PAGE loading buffer (Beyotime) was transferred to a fresh $1.5 \mathrm{~mL}$ microfuge tube, serving as a input control. The rest sample was incubated with $1 \mu \mathrm{L}$ primary antibody or isotype-matched IgG overnight at $4^{\circ} \mathrm{C}$ with slow-speed rotating. Protein G Agarose beads (20398, ThermoFisher Scientific) were also incubated with $4 \%$ bovine serum albumin for blockade overnight at $4^{\circ} \mathrm{C}$ with slow-speed rotating. Samples added with equal volume of beads were again slow-speed rotated at room temperature (RT) for 1 hour, and then washed 3-4 times with phosphate buffered saline (PBS) with highspeed rotation. The samples were centrifuged at $1000 \times \mathrm{g}$ for $2 \mathrm{~min}$ and PBS was completely removed. The samples were mixed with $30 \mu \mathrm{L}$ SDS sample buffer and heated at $95^{\circ} \mathrm{C}$ for $5 \mathrm{~min}$, and then detected by western blot analysis as described above.

\section{Immunofluorescence}

Cells planted in 8-well chamber slides (154534, Nalge Nunc International) were fixed with $4 \%$ formaldehyde (BL539A, Biosharp) at room temperature for $20 \mathrm{~min}$. Cells were washed twice with PBS, and then permeabilized and blocked with PBS buffer containing 0.2\% Triton X-100\% and $1 \%$ BSA at RT for 1 hour, and then incubated with primary antibodies overnight at $4^{\circ} \mathrm{C}$. Cells were washed twice with PBS and then incubated with secondary antibodies at room temperature for $20 \mathrm{~min}$, protected from light. Nuclei were stained with DAPI (0100-20, SouthernBiotech). The slides were observed using Zeiss LSM710 confocal microscope. The quantification of fluorescence intensity and the co-localization was analyzed by Image software (V.2.0.0-rc-69/1.52p).

\section{ER and Golgi apparatus fractions enrichment}

Minute ER Enrichment Kit (ER-036, Invent) and Minute Golgi apparatus Enrichment Kit (GO-037, Invent) were used to extract protein fractions from the ER and Golgi apparatus, respectively. The experiments were performed according to the manufacturer's instructions. Protein concentration was determined by BCA Protein Assay Kit (23225, ThermoFisher Scientific).

\section{Protein purification and ELISA}

THADA-His vector was purchased from General Biosystem and constructed as described above. Proteins were expressed individually in Transetta (DE3) cells grown in LB medium with shaking at $220 \mathrm{rpm}, 37^{\circ} \mathrm{C}$. Overproduction of the protein was then induced by isopropylthio- $\beta$-galactoside (IPTG) to a final concentration of $0.2 \mathrm{mM}$ at $\log$ phase. After grown for an additional 14 hours at $30^{\circ} \mathrm{C}$, the cells were harvested by centrifugation at $6000 \times \mathrm{g}$ for $10 \mathrm{~min}$. The bacterial cells were lysed via high pressure homogenizer in buffer containing $20 \mathrm{mM}$ Tris-HCl $(\mathrm{pH}$ $8.0), 300 \mathrm{mM}$ NaCl, $1 \mathrm{mM}$ PMSF, $5 \mu \mathrm{g} / \mathrm{mL}$ DNase, $2 \mathrm{mM}$ $\mathrm{MgCl}_{2}$. The THADA protein was purified on Ni-NTA resin (Smart Life Sciences) followed by the application of anion exchange column (HiTrap Capto Q ImpRes, $5 \mathrm{~mL}$ ) and size exclusion chromatography (Superdex 200 
Increase 10/300 GL). Purified protein was concentrated and exchanged into PBS and stored at $-80^{\circ} \mathrm{C}$.

For ELISA, $2-16 \mu \mathrm{g} / \mathrm{mL}$ of THADA was added to a 96-well ELISA plate and incubated overnight at $4^{\circ} \mathrm{C}$. Following five washes with PBST, non-specific binding sites were blocked with $2 \%$ BSA in PBS; $1 \mu \mathrm{g} / \mathrm{mL}$ PD-L1-Fc was added to the wells followed by antihuman IgG HRP (Jackson Immuno Research). Enzyme detection was achieved using Pierce TMB Substrate Kit (Thermo Scientific) and signal intensities were quantified using the Thermo Scientific Multiskan FC.

\section{Analysis of PD-L1 degradation rate}

Cells transfected with THADA siRNAs were co-incubated with cycloheximide (C104450, Sigma) $(50 \mu \mathrm{g} / \mathrm{mL})$ at the indicated time points, and subjected to western blot analysis. The intensity of each band was quantified by measuring gray values with the same-sized frame after subtracting background using ImageJ software (V.2.0.0rc-69/1.52p). The relative PD-L1 intensity of each lane was normalized by the corresponding GAPDH intensity and the intensity at time point ' 0 '.

\section{PD-1-binding assay by fluorescence activated cell sorting}

Cells were harvested by trypsin digestion and centrifuged at $1500 \mathrm{rpm}$ for $5 \mathrm{~min}$. The pellet resuspended and washed twice with PBS was then incubated with recombinant human PD-1 FC chimera protein (1086-PD-050, R\&D Systems, 1:80 dilution) in $200 \mu \mathrm{L}$ staining buffer per tube at RT for 1 hour. The pellet was washed twice with staining buffer and incubated with antihuman Alexa Fluor 488 dye conjugated antibody (A-11013, ThermoFisher Scientific, 1:400 dilution) at RT for $30 \mathrm{~min}$, protected from light. The pellet was then washed twice with staining buffer and suspended with $300 \mu \mathrm{L}$ staining buffer and examined by fluorescence activated cell sorting (FACS) in fluorescein isothiocyanate channel. The results were analyzed by FlowJo software.

\section{T cell killing assay}

RKO cells transfected with THADA siRNAs or negative control siRNAs were cultured for 48 hours and re-seeded into a 96-well plate with $1 \times 10^{6}$ cells per well. Human peripheral blood mononuclear cells (PBMC, CE-003, StemEry) activated by $100 \mathrm{ng} / \mathrm{mL}$ CD3 antibody (317303, BioLegend), $100 \mathrm{ng} / \mathrm{mL}$ CD28 antibody (302913, BioLegend) and $10 \mathrm{ng} / \mathrm{mL}$ IL2 (589102, BioLegend) were co-cultured with RKO cells at $10: 1$ ratio at $37^{\circ} \mathrm{C}$ under $5 \% \mathrm{CO}_{2}$ in RPMI-1640 complete medium. About 12 hours later, the cells were stained with fluorescent dye caspase-3/7 (I35106, ThermoFisher Scientific) for 1 hour and then Hoechst for 5 min according to the manufacturer's instructions. The cells were washed twice and examined by fluorescence microscopy.

\section{Immunohistochemistry staining and CRC tissue microarrays}

Tissues samples were deparaffinized and rehydrated, and the antigen retrieval was carried out in citrate antigen retrieval solution. After blocking endogenous peroxidase with $3 \% \mathrm{H}_{2} \mathrm{O}_{2}$ for $15 \mathrm{~min}$ and blocking with goat serum for 1 hour, tissue samples were incubated with primary antibodies overnight at $4^{\circ} \mathrm{C}$, followed by incubation with biotin-conjugated secondary antibody at RT for 1 hour. $\mathrm{DAB}$ was used as chromogen and nuclei were counterstained with hematoxylin. CRC tissue microarray was purchased from BioChip (Shanghai, China) with informed patient consent provided. The tissue microarray involved matched normal tissues as well as primary and metastatic tumor tissue samples from 22 patients (more than one metastatic tumor in some of the patients). Immunohistochemistry (IHC) staining was assessed using $\mathrm{H}$ score with the formula (weak) $\% \times 1+($ moderate $) \% \times 2+($ strong $) \% \times 3$, ranging from 0 to 300 .

\section{Statistical analysis}

Column bar graphs and scatter plots were plotted using GraphPad Prism V.8. Values were mean \pm SD from three independent experiments. Two-sided Student's t-test was applied for the comparison of two independent samples and one-way analysis of variance with post hoc test (Tukey) was applied to compare more than two groups. ImageJ (V.2.0.0-rc-69/1.52p) was applied for the data quantification of immunofluorescence and western blot analysis. The average fluorescence intensity was means \pm SD. from three independent experiments, and each individual dot represented the average fluorescence intensity of three fields in each replicate. The co-localization factor (Pearson's R value) were calculated using Image J (V.2.0.0-rc-69/1.52p) with the plugin 'coloc2' to evaluate the co-localization between two proteins. Values are means \pm SD from three independent experiments, and each individual dot represents the average co-localization coefficiency of three fields in each replicate. The number of samples assigned to each treatment was selected to provide sufficient statistical power to discern significant differences in different groups, based on prior experience with the experiment. The only data points excluded were those that were clear outliers due to technical problems in assays done in triplicate experiments. In this work, $p$ value $<0.05$ was considered to be statistically significant.

\section{RESULTS \\ Identification of THADA as a positive regulator of PD-L1}

To find potential regulators of PD-L1, the combined analyses of genomes and proteomics were applied. It has been established that genes with related functions are often altered in cancer genomes in certain patterns, such as 'co-occurrence' or 'mutual exclusivity'. To this end, we assessed the associations of the copy number alterations (CNAs) between the PD-L1 (CD274) gene and a series of low-confidence interactors of PD-L1 listed in the BioGrid database using the CRC data set summarized in the Cancer Genome Atlas (TCGA) (figure 1A). As a result, we found several PD-L1 putative interactors had significant associations with PD-L1 in their CNAs. After excluding the proteins that had been reported to 


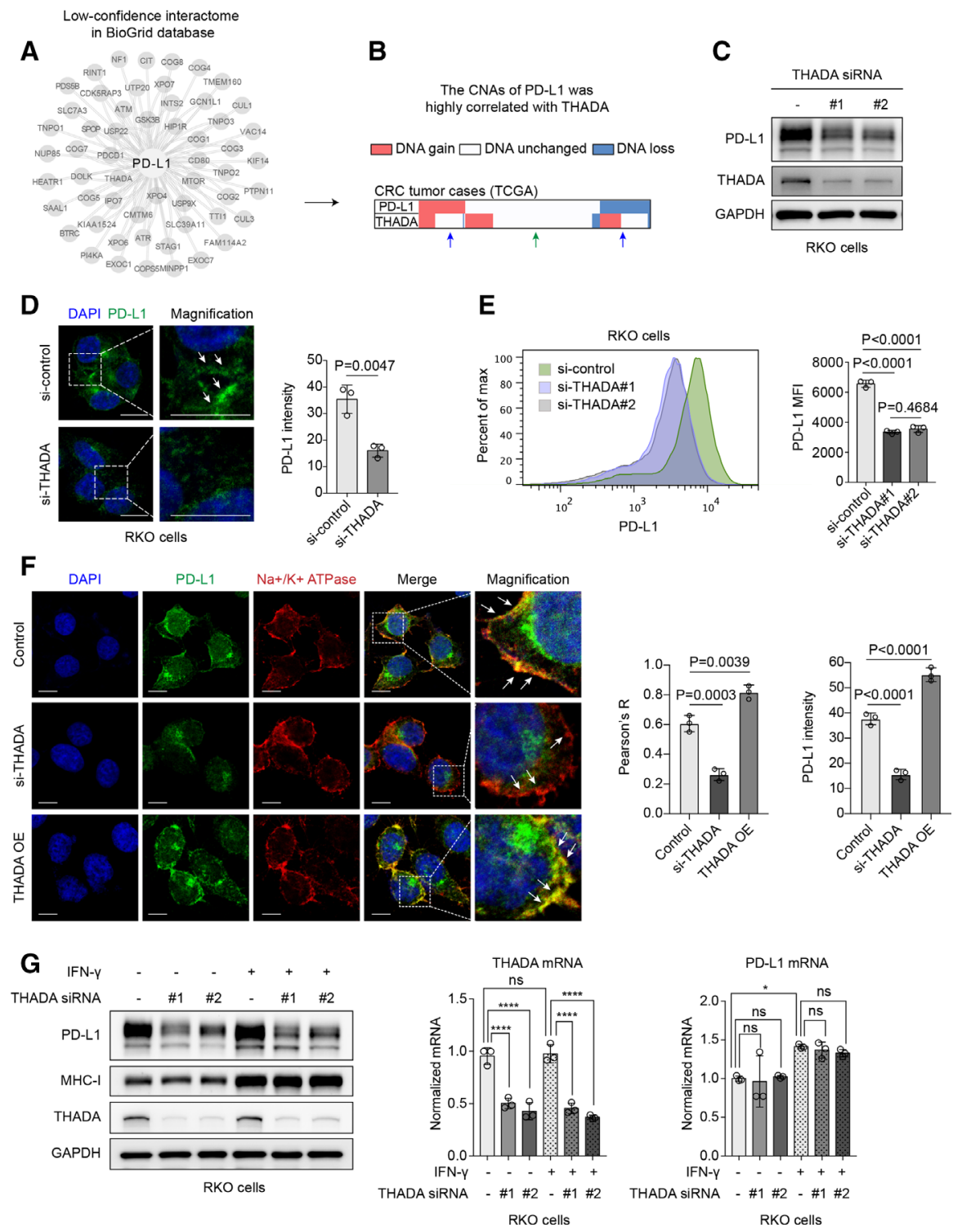

Figure 1 Thyroid adenoma associated gene (THADA) positively regulated programmed death-ligand 1 (PD-L1). Identification of THADA as a putative regulator of PD-L1. Combined analysis of a set of low-confidence interactors of PD-L1 summarized in the BioGrid database (A) with their gene copy number alterations (CNAs) in the colorectal cancer (CRC) data set of TCGA. The CNAs of THADA and PD-L1 were associated in the TCGA CRC data set (B). In CRC cases with no alteration in copy number of PD-L1 gene, THADA gene also tended to keep unchanged (green arrow), while in tumors with gained or loss of PD-L1 gene, THADA gene was prone to be amplified or diminished as well (blue arrow). (C) Western blot analysis showing the effect of THADA depletion by two distinct small interfering RNAs (siRNAs) on PD-L1 expression in RKO cells. The experiments were repeated three times independently with similar results. (D) Left, immunofluorescence assay showing RKO cells transfected with THADA siRNAs. Green, PD-L1; blue, DAPI nucleus staining. Scale bars, $10 \mu \mathrm{m}$. White dashed boxes indicate the representative fields to be magnified. Right, quantification of fluorescent intensity of PD-L1. Values are means \pm SD from $n=3$ independent experiments. Statistical differences were evaluated by two-sided Student's t-test. (E) Left, flow cytometry showing the effect of THADA knockdown by two distinct siRNAs on PD-L1. Right, quantification of mean fluorescence intensity (MFI) of PD$L 1$. Values are means $\pm S D$ from $n=3$ independent experiments. Statistical differences were evaluated by analysis of variance (ANOVA) post hoc test (Tukey). (F) Immunofluorescence assay showing PD-L1 localization on the plasma membrane in THADA depleted or overexpressed RKO cells. Scale bars, $10 \mu \mathrm{m}$. White dashed boxes indicate the representative fields to be magnified. Values are means $\pm S D$ from $n=3$ independent experiments. Statistical differences were evaluated by ANOVA post hoc test (Tukey). (G) RKO cells transfected with two distinct THADA siRNAs and co-incubated with interferon (IFN)- $\gamma(100 \mathrm{ng} / \mathrm{mL}$, 24 hours), subjected to western blot analysis (left) and RT-PCR (right). Values are means \pm SD from $n=3$ independent experiments. Statistical differences were evaluated by ANOVA post hoc test (Tukey). ${ }^{*} \mathrm{P}<0.033$; ${ }^{* \star \star *} \mathrm{P}<0.0001$; ns, no significance.

be related to PD-1/PD-L1 axis such as CDK5RAP3 and CD80, ${ }^{46-48}$ our previous work had characterized HIP1R as a potent suppressor of PD-L1 that could deliver PD-L1 to lysosomal degradation..$^{25}$ Meanwhile, we noticed that the CNAs of THADA and PD-L1 were also associated (figure 1B). In CRC cases with no alteration in copy 
number of PD-L1 gene, THADA gene was prone to keep unchanged (figure 1B, green arrow), while in tumor cases with gained or loss of PD-L1 gene, THADA gene tended to be amplified or diminished as well (figure 1B, blue arrow). THADA gene has rarely been studied. Although several studies have correlated THADA with an increased risk of multiple cancers, ${ }^{49-52}$ the molecular mechanism remains poorly understood. Thus, we sought to investigate whether THADA had regulatory effect on PD-L1.

First, we probed whether THADA affected PD-L1 expression. Human CRC RKO, LoVo and HCT116 cells were, respectively, transfected with ectopic Myc-tagged THADA to examine the expression of PD-L1. Western blot analysis revealed that overexpression of THADA markedly enhanced the expression of PD-L1 in all the above cells (online supplemental figure 1A). On the contrary, depletion of THADA by specific siRNAs substantially decreased PD-L1 expression in the same cell lines (figure $1 \mathrm{C}$ and online supplemental figure 1B). The effect of THADA on PD-L1 was also validated with similar results in other human tumor cell lines such as breast cancer MDA-MB231 cells and melanoma A375 cells (online supplemental figure $1 \mathrm{C}, \mathrm{D})$, suggesting a widespread regulatory effect of THADA on PD-L1 in various cancers. Immunofluorescence assays (figure 1D) and flow cytometry (figure 1E) also revealed that THADA depletion caused a reduction of PD-L1. Generally, PD-L1 proteins exert their function on the tumor cell membrane. To this end, we examined the co-localization of PD-L1 and the plasma membrane under immunofluorescence microscopy. Results revealed that THADA knockdown significantly reduced the distribution of PD-L1 on the plasma membrane and the fluorescence intensity of PD-L1, while overexpression of THADA abundantly increased the plasma membrane localization of PD-L1 as well as the intensity of PD-L1 (figure 1F, white arrow). These data collectively demonstrate that THADA has significant positive effect on PD-L1 expression in multiple cancer cells.

To examine whether the regulation of PD-L1 by THADA was at the mRNA or the protein level, we established HCT116 CRC cells with low expression of endogenous PD-L1 that stably expressed ectopic Flag-tagged PD-L1. Western blot analysis revealed that overexpression or depletion of THADA, respectively, upregulated or downregulated exogenous PD-L1 in HCT116 ectopic PD-L1 stable cells (online supplemental figure 1E,F). As IFN- $\gamma$ was a potent inducer of PD-L1 upregulation by activating JAK/STAT/IRF axis, ${ }^{53}$ tumor cells transfected with THADA siRNAs were also treated with IFN- $\gamma$ to examine whether the effect of THADA on PD-L1 was dependent on IFN- $\gamma$. As a result, western blot analysis and RT-PCR revealed that depletion of THADA suppressed both constitutive and IFN- $\gamma$-induced PD-L1 protein but displayed no effect on PD-L1 mRNA expression (figure $1 \mathrm{G}$ and online supplemental figure 1G). Particularly, knockdown of THADA did not impair the expression of MHC-I in the presence or absence of IFN- $\gamma$. Interestingly, no change in THADA mRNA or protein expression was observed under IFN- $\gamma$ treatment. Taken together, these findings indicate THADA as a distinct positive regulator that selectively modulates PD-L1 protein but not mRNA expression in diverse cancer cells.

\section{THADA interacted with PD-L1 to maintain its Golgi residency}

Next, we validated how THADA impacted on PD-L1 protein expression. In consideration of the data developed from high-throughput affinity-purification mass spectrometry, which indicated THADA as a putative interactor of PD-L1, ${ }^{54} 55$ we speculated that THADA might exert effect by directly interacting with PD-L1. A strong interaction of endogenous PD-L1 and THADA was observed in RKO cells by Duolink assay under fluorescence microscopy, while depletion of THADA substantially decreased the binding (figure 2A). Co-IP assays revealed that THADA could physically bind to endogenous PD-L1 in RKO and LoVo cells (figure 2B) and exogenous PD-L1 in HCT116 ectopic PD-L1 stable cells (figure 2C). The purified THADA-his protein was immobilized onto the ELISA plates, and purified PD-L1-FC was added to bind THADA. The results confirmed direct interaction between both purified proteins (figure 2D). Immunofluorescence assays also showed the co-localization of THADA and PD-L1 in RKO cells (figure 2E,F). Intriguingly, PD-L1 showed the localization to the perinuclear clusters in the control group (figure 2E, white arrow), while a more diffuse appearance around the nucleus in THADAdepleted group (figure 2F, white arrow), suggesting THADA might affect the intracellular distribution of PD-L1. Since PD-L1 protein goes through the secretory pathway via the ER and the Golgi apparatus in sequence for maturation before it functions on the cell surface, we speculated the perinuclear region where PD-L1 stayed in the control group was the Golgi apparatus while the more diffuse region around the nucleus in THADA-depleted group was the ER. To this end, we tested the localization of PD-L1 to the ER and Golgi, respectively, in THADA-knockdown RKO cells. As expected, depletion of THADA markedly increased the localization of PD-L1 to the ER (GRP94), while decreased the distribution of PD-L1 to the Golgi (58K) (figure 2G-I). To further explore the effect of THADA on PD-L1 distribution, total protein fractions, ER protein fractions as well as Golgi protein fractions were extracted, respectively, from RKO cells transfected with THADA siRNAs or negative control siRNA. Western blot analysis revealed that with MG132 treatment, THADA depletion caused an appreciable reduction of PD-L1 expression in the Golgi rather than the ER (figure 2J). These findings collectively suggest that THADA can interact with PD-L1 and maintains its Golgi residency, while silencing THADA significantly leads to ER retention of PD-L1 and PD-L1 exhaustion in the Golgi apparatus. 

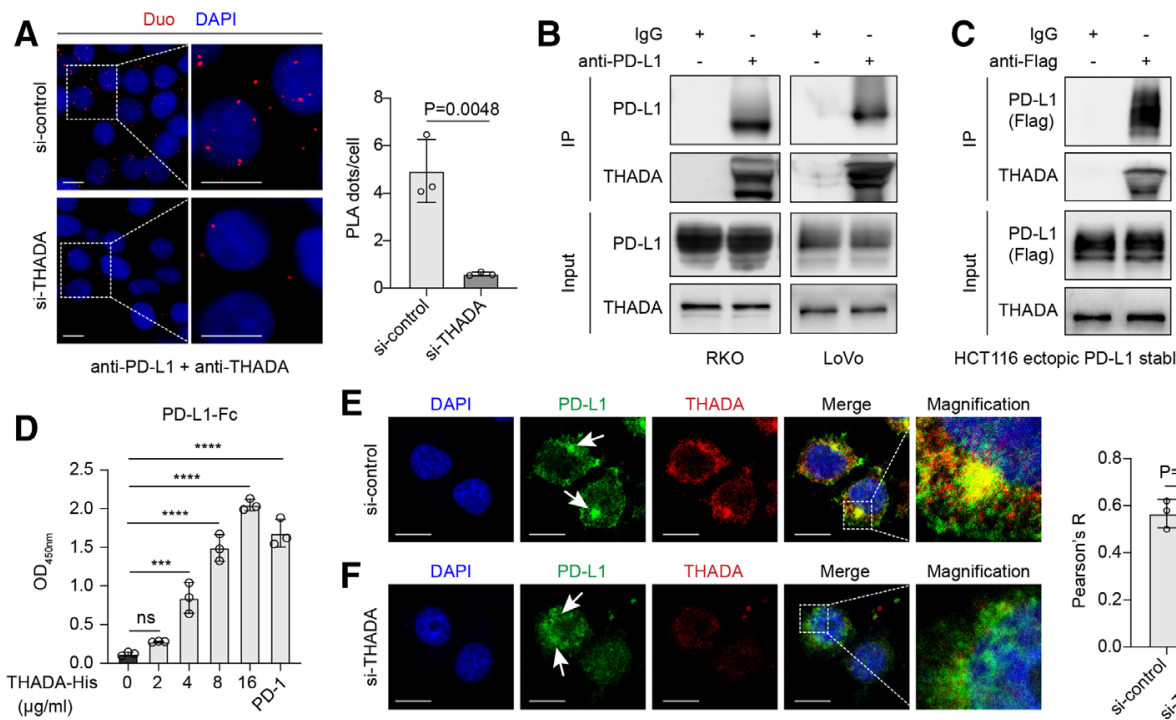

HCT116 ectopic PD-L1 stable cells
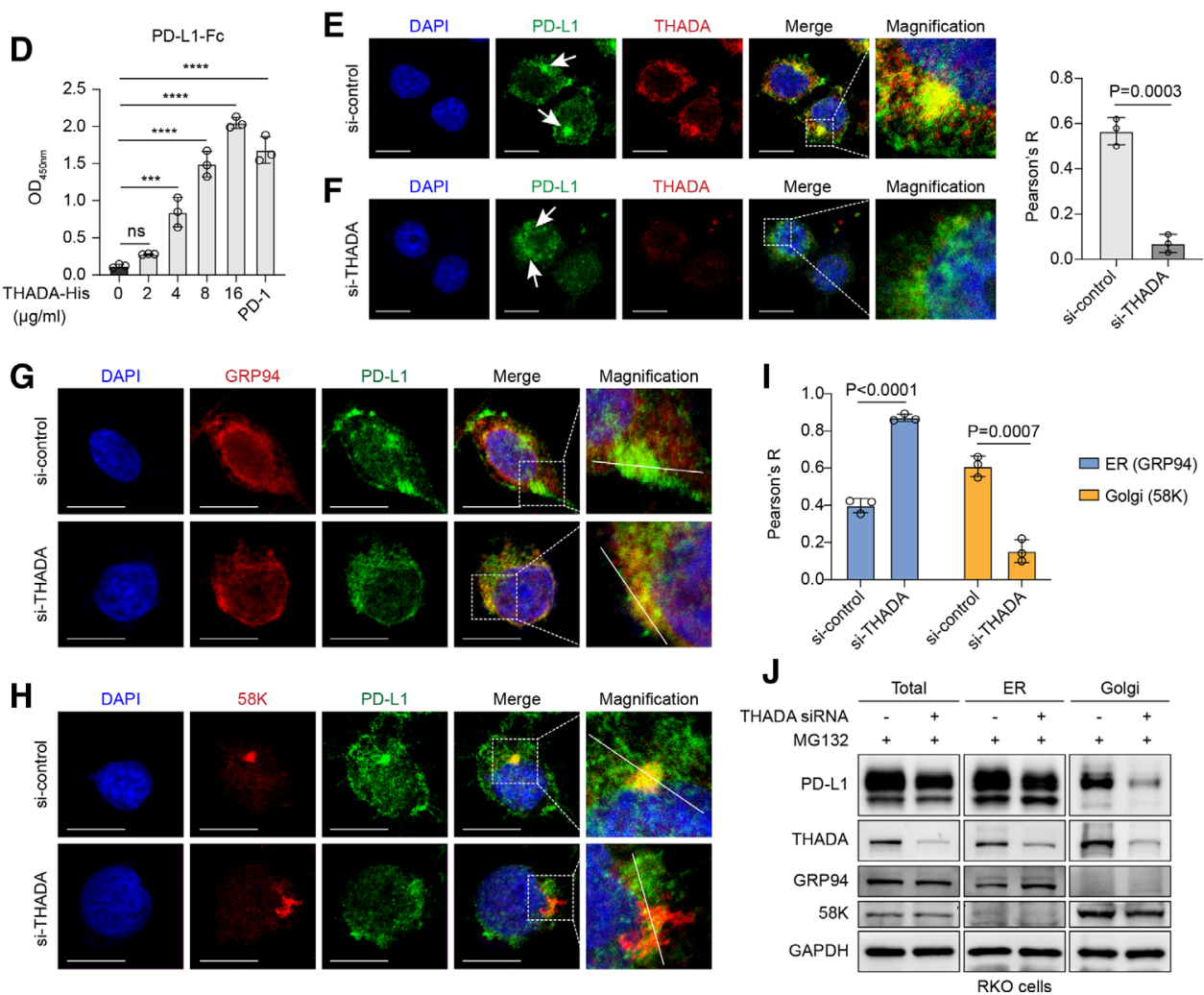

Figure 2 Thyroid adenoma associated gene (THADA) interacted with programmed death-ligand 1 (PD-L1) to maintain its Golgi residency. (A) Duolink assay showing the interaction of endogenous THADA and PD-L1 proteins in RKO cells. Red dots indicating the binding of two proteins. Nuclei were stained with DAPI. Scale bar, $10 \mu \mathrm{m}$. Values are means $\pm S D$ from three random fields in each treatment. Statistical differences were evaluated by two-sided Student's t-test. Co-immunoprecipitation (Co-IP) assay detecting the interaction between THADA and endogenous PD-L1 (B) and exogenous PD-L1 (C), respectively, in the indicated cells. The experiments were repeated three times independently with similar results. (D) ELISA showing the direct interaction between purified proteins of THADA-His and PD-L1-FC $(1 \mu \mathrm{g} / \mathrm{mL})$. Programmed cell death 1 (PD-1) was served as a positive control $(1 \mu \mathrm{g} / \mathrm{mL})$. Statistical differences were evaluated by analysis of variance (ANOVA) post hoc test (Tukey). ${ }^{* * *} \mathrm{P}=0.0001$; ${ }^{* * * *} \mathrm{p}<0.0001$; ns, no significance. (E, F) Immunofluorescence assays showing the co-localization of PDL1 and THADA in RKO cells with indicated treatment. Green, PD-L1; red, THADA; blue, DAPI nucleus staining. Scale bars, $10 \mu \mathrm{m}$. Values are means $\pm S D$ from $n=3$ independent experiments. The $p$ values were evaluated by two-sided Student's t-test. Immunofluorescence assays showing the co-localization of PD-L1 and GRP94 (G)/58K (H) in RKO cells transfected with THADA small interfering RNAs (siRNAs). Scale bars, $10 \mu \mathrm{m}$. White dashed boxes denote the representative fields to be magnified. (I) Quantification of the co-localization between PD-L1 and endoplasmic reticulum (ER)(GRP94)/Golgi(58K) in RKO cells. Values are means $\pm S D$ from $n=3$ independent experiments. The $p$ values were evaluated by two-sided Student's t-test. $(J)$ Western blot analyses showing the effect of THADA depletion on PD-L1 proteins extracted from total cell, the ER and Golgi apparatus with MG132 treatment $(10 \mu \mathrm{M}, 6$ hours).

\section{THADA was required for Sec24A-dependent COPII vesicle trafficking of PD-L1}

In eukaryotes, protein transport from the ER to the Golgi apparatus is dominated by COPII-coated vesicles. By analyzing a set of interactors of THADA summarized in the BioGrid database, we found THADA was identified to interact with COPII major components
Sec23A and Sec24A using quantitative tandem mass spectrometry $^{56}$ (figure 3A). Sec23/Sec24 heterodimer forms the inner shell of the COPII coat and Sec24 is the sole module of five core COPII components that can specifically recognize and interact with cargo proteins, thereby administering the exact cargo export from the ER. ${ }^{27}{ }^{32}$ Sec24A is one of the four isoforms 

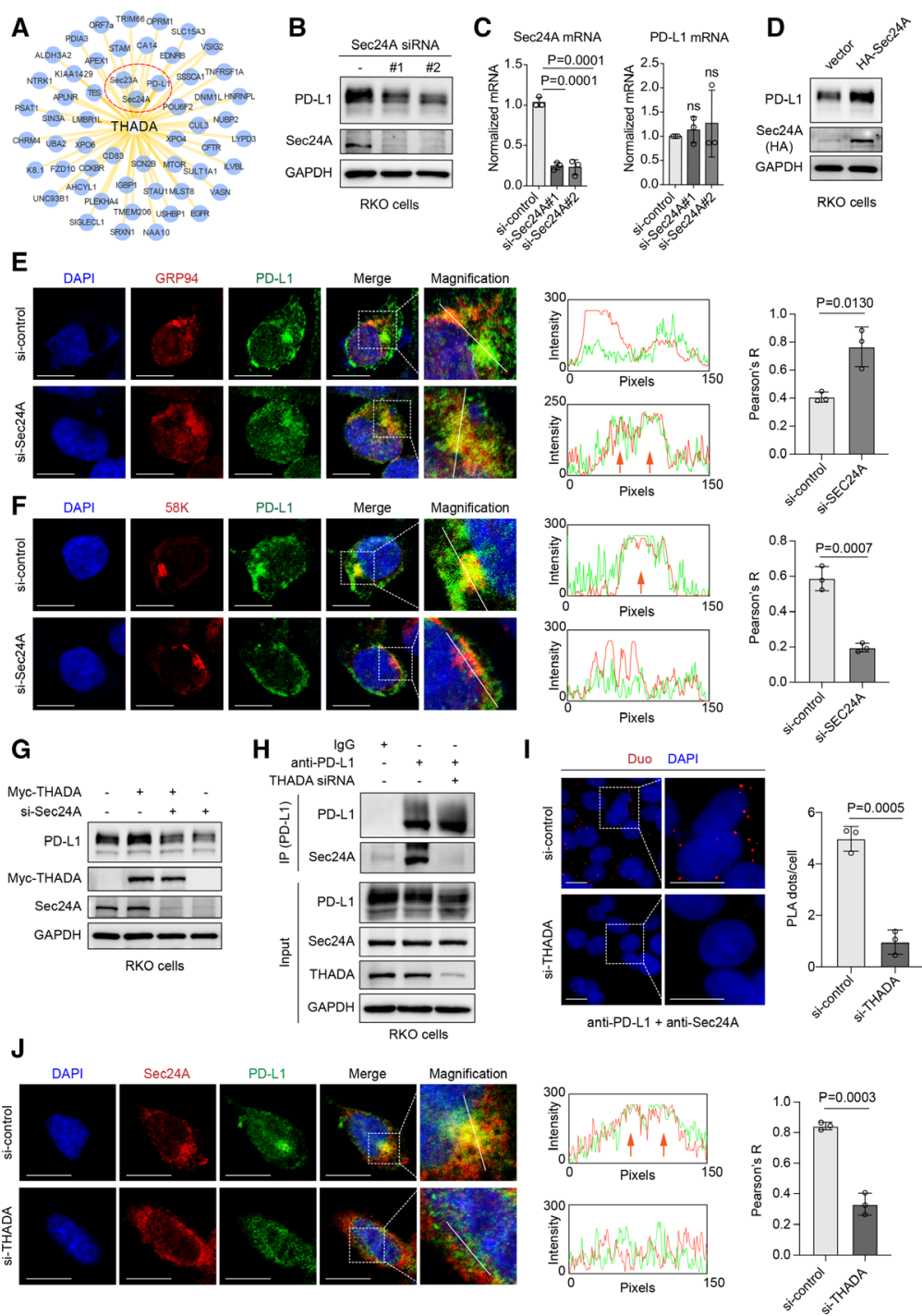

Figure 3 Thyroid adenoma associated gene (THADA) required for Sec24A-dependent vesicle trafficking of programmed death-ligand 1 (PD-L1). (A) A set of low-confidence interactors of THADA summarized in the BioGrid database. RKO cells transfected with two distinct Sec24A small interfering RNAs (siRNAs) and subjected to western blot analysis (B) and RT-PCR $(C)$, respectively. Values are mean $\pm S D$ from $n=3$ independent experiments. Statistical differences were evaluated by analysis of variance (ANOVA) post hoc test (Tukey); ns, no significance. The experiments were repeated three times independently with similar results. (D) Western blot analyses showing the effect of Sec24A overexpression (HA-Sec24A) on PD-L1 in RKO cells. The experiment was repeated three times independently with similar results. Left, immunofluorescence assays showing the co-localization between PD-L1 and GRP94 (E)/58K (F) in RKO cells transfected with Sec24A siRNAs. Scale bars, $10 \mu \mathrm{m}$. White dashed boxes denote the representative fields to be magnified. Middle, the intensity profiles of PD-L1 and GRP94 $(\mathrm{E}) / 58 \mathrm{~K}(\mathrm{~F})$ along the white line. Orange arrows denote the co-localization. Right, values are means $\pm \mathrm{SD}$ from $\mathrm{n}=3$ independent experiments. The $p$ values were evaluated by two-sided Student's t-test. (G) RKO cells transfected Myc-tagged THADA and Sec24A siRNAs as indicated and subjected to western blot analysis with indicated antibodies. (H) Co-immunoprecipitation (CoIP) assay showing the interaction between PD-L1 and Sec24A in RKO cells transfected with THADA siRNAs. The experiments were repeated three times independently with similar results. (I) Duolink assay showing the interaction of endogenous PD-L1 and Sec24A proteins in RKO cells. Red dots indicating the binding of two proteins. Nuclei were stained with DAPI. Scale bar, $10 \mu \mathrm{m}$. Values are means \pm SD from three random fields in each treatment. Statistical differences were evaluated by two-sided Student's t-test. (J) Left, immunofluorescence assay showing the co-localization between PD-L1 and Sec24A in RKO cells transfected with THADA siRNAs. Scale bars, $10 \mu \mathrm{m}$. White dashed boxes denote the representative fields to be magnified. Middle, the intensity profiles of PD-L1 and Sec24A along the white line. Orange arrows denote the co-localization. Right, values are means $\pm S D$ from $n=3$ independent experiments. The $p$ values were evaluated by two-sided Student's t-test.

of human Sec24 family, whereas the respective role of each isoform remains unclear. Previous studies have identified some proteins as the cargo adaptors to couple particular transmembrane cargo to COPII modules. Thus, such cargoes require a dual interaction with both the cargo adaptor and Sec24 to drive COPII vesicle trafficking. ${ }^{28} 374057$ Given the results that THADA notably affected PD-L1 distribution in 
the ER and Golgi, we asked whether THADA played a role in such COPII-associated transport of PD-L1.

First, we sought to test Sec24A was responsible for the COPII transport of PD-L1. RKO cells treated with Sec24A siRNAs were subjected to western blot analysis and RT-PCR. Results revealed that Sec24A depletion substantially reduced endogenously expressed PD-L1 protein (figure 3B) without affecting PD-L1 mRNA expression (figure 3C). Similar results were observed in exogenous PD-L1 in HCT116 ectopic PD-L1 stable cells (online supplemental figure 2A). On the contrary, ectopic expression of Sec24A caused an increase in PD-L1 expression (figure 3D). Immunofluorescence assays were performed to detect PD-L1 distribution in the ER and Golgi in Sec24A-depleted RKO cells. In consistent with our previous results in figure 2G-I, silencing Sec24A significantly facilitated PD-L1 retention in the ER (GRP94, figure 3E) while blocked PD-L1 distribution in the Golgi (58K, figure 3f). These findings indicate Sec24A participates in PD-L1 trafficking from the ER to the Golgi apparatus.

Next, we investigated whether THADA was involved in such Sec24A-mediated trafficking mechanism. Co-IP assays characterized the interaction of THADA and Sec24A (online supplemental figure 2B,C) as well as the interactions among endogenously expressed THADA, Sec24A and PD-L1 (online supplemental figure 2D). To determine whether THADA-mediated upregulation of PD-L1 was associated with Sec24A, we silenced Sec24A in THADA-overexpressing cells and tested PD-L1 expression by western blot analyses. Results revealed that THADAinduced upregulation of both endogenous and exogenous PD-L1 could be effectively blocked by the absence of Sec24A (figure 3G and online supplementary figure 2E), indicating the effect of THADA on PD-L1 was dependent on Sec24A. Correspondingly, to verify whether THADA was also indispensable for the interaction between PD-L1 and Sec24A, we examined the binding of PD-L1 and Sec24A in the presence or absence of THADA. Co-IP assays (figure $3 \mathrm{H}$ ) and Duolink assays (figure 3I) both showed an appreciable interaction of PD-L1 and Sec24A, while THADA knockdown substantially suppressed the association. The intracellular localization of PD-L1 and Sec24A was examined by immunofluorescence assays. The results identified that depletion of THADA strikingly decreased the co-localization of PD-L1 and Sec24A (figure 3J). Taken together, these findings indicate THADA as an indispensable intermediator of PD-L1 and Sec24A, thereby manipulating the PD-L1-specific vesicle trafficking from the ER to the Golgi complex.

\section{Depletion of THADA induced PD-L1 degradation via ERAD pathway}

As depletion of THADA markedly accelerated the degradation rate of PD-L1 protein (figure 4A and online supplemental figure $3 \mathrm{~A}$ ), we sought to investigate how PD-L1 was eliminated in the absence of THADA. A previous study indicated that the glycosylation-deficient
PD-L1 trapped in the ER lumen could be targeted for ERAD degradation. ${ }^{18}$ Given our above results that THADA depletion significantly caused ER retention of PD-L1, we asked whether PD-L1 detained in the ER lumen would serve as ERAD substrates, thereby recognized and extracted into the cytoplasm by ERAD complex for proteasomal degradation. To confirm the hypothesis, tumor cells transfected with THADA siRNAs were co-incubated with the proteasome inhibitor MG132, ERAD inhibitor Eer I and lysosomal inhibitor CQ, respectively, and subjected to western blot analysis. Results revealed that MG132 (figure 4B and online supplemental figure 3B) and Eer I (figure 4C and online supplemental figure 3C) both appreciably restored THADA-induced reduction of PD-L1 in a time-dependent manner, while CQ displayed no effect (figure 4D). Additionally, a substantial increase of PD-L1 ubiquitination was found in THADAknockdown CRC cells by Co-IP assays (figure 4E,F).

HRD1 complex is the major ERAD complex in mammalians containing E3 ubiquitin ligase HRD1 and the lectinlike proteins OS-9 and XTP3-B that recognize and interact with ERAD substrates for degradation. ${ }^{43-45}$ OS-9 and XTP3-B are the human homologues of Yos9. Although several studies have demonstrated functional redundancy between OS-9 and XTP3-B, the exact roles of the two proteins remain poorly understood ${ }^{58-60}$ In a recent study, HRD1, OS-9 and XTP3-B were indicated to bind PD-L1 and modulate its ERAD degradation..$^{18}$ To this end, HRD1, OS-9 and XTP3-B were knocked down by three distinct siRNAs, respectively, in THADA-depleted tumor cells. As a result, depletion of XTP3-B and HRD1 could respectively restore THADA-mediated downregulation of PD-L1 in RKO cells (figure 4G and online supplemental figure 3D). However, OS-9, the homologue of XTP3-B, showed less effect on PD-L1 expression. Similar results were observed in exogenous PD-L1 in HCT116 ectopic PD-L1 stable cells (figure 4H). Co-IP assays revealed that the binding of both endogenous and exogenous PD-L1 to XTP3-B and HRD1 was considerably increased when tumor cells were treated with THADA siRNAs (figure 4I,J). These data collectively suggest that silencing THADA eliminates PD-L1 by promoting PD-L1 capture by HRD1 complex via ERAD pathway, ultimately contributing to PD-L1 degradation by the ubiquitin-proteasome system (scheme in figure $4 \mathrm{~K}$ ).

\section{THADA knockdown promoted T cell-mediated antitumor immunity}

Several studies have associated THADA with a risk factor for multiple cancers, although the underlying mechanism remains unclear. ${ }^{49-52}$ As the above results showed THADA could effectively stabilize PD-L1 expression in tumor cells, we aimed to evaluate the antitumor effect of THADA.

As our previous study had assessed PD-L1 expression in CRC tissues ${ }^{61}$ we sought to examine the prevalence of THADA and the relationship between THADA and PD-L1 expression in tumor tissues using the same CRC 
A
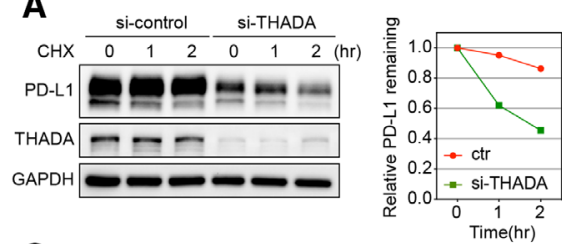

C si-control si-THADA
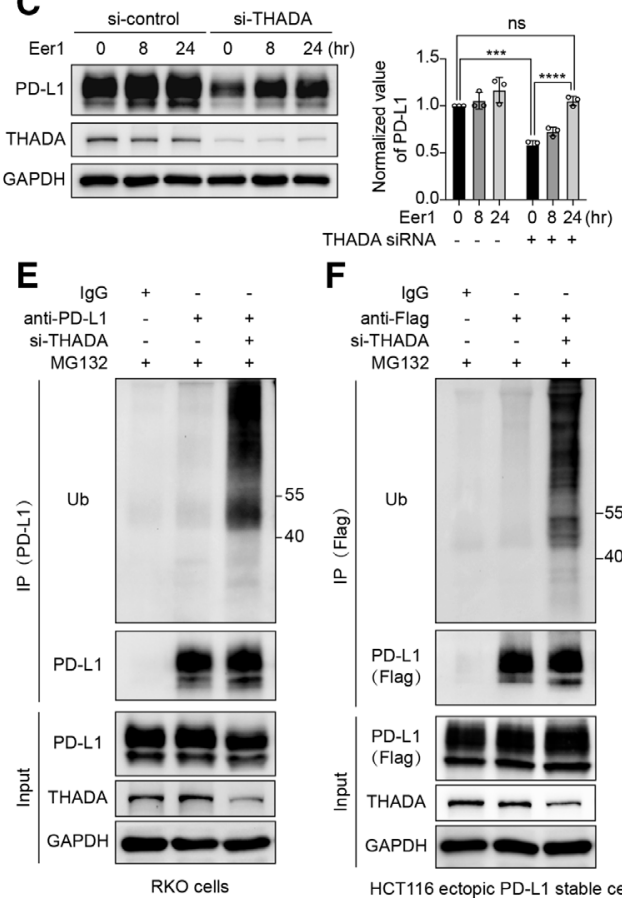

F
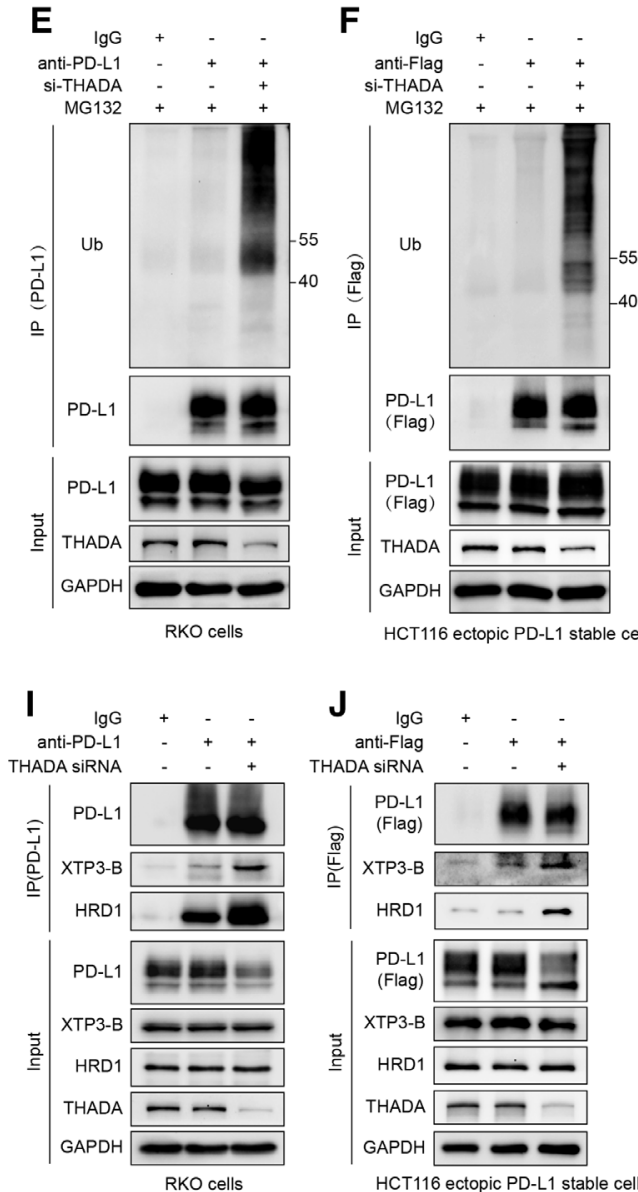
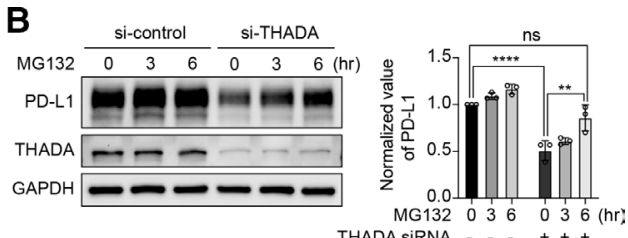

D

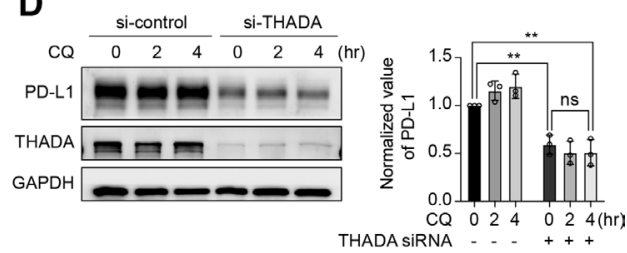

G

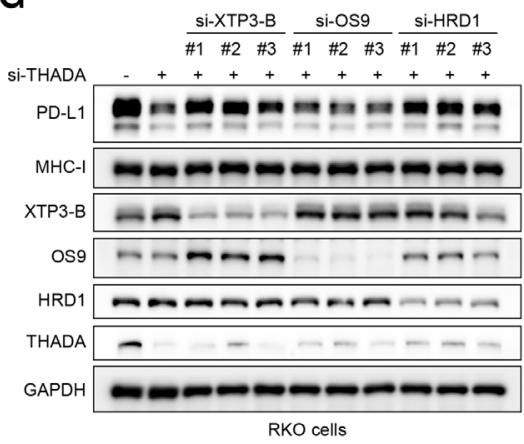

H
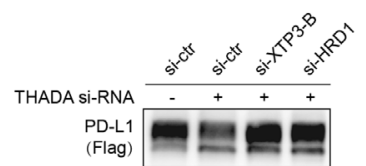

THADA - - -

ХтРз-в ---

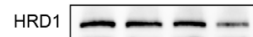

GAPDH --

K

HCT116 ectopic PD-L1 stable cells

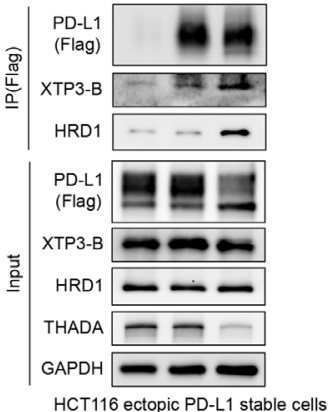

Figure 4 Thyroid adenoma associated gene (THADA) depletion induced endoplasmic reticulum (ER)-associated degradation of programmed death-ligand 1 (PD-L1). (A) Left, RKO cells transfected with THADA small interfering RNAs (siRNAs) and coincubated with cycloheximide $(\mathrm{CHX})(50 \mu \mathrm{g} / \mathrm{mL})$ for denoted time points, and subjected to western blot analysis with indicated antibodies. Right, quantification of gray value of remained PD-L1. The experiment was repeated three times independently with similar results. Left, western blot analysis showing the effect of THADA depletion on PD-L1 expression in the absence or presence of proteasomal inhibitor MG-132 $(10 \mu \mathrm{M})(B)$, endoplasmic reticulum-associated degradation (ERAD) inhibitor eeyarestatin I (Eer I) $(10 \mu \mathrm{M})(C)$, lysosomal inhibitor chloroquine $(C Q)(25 \mu M)(D)$ in LoVo cells. Right, values are means $\pm S D$ from three independent experiments. Statistical differences were determined by analysis of variance (ANOVA) post hoc test (Tukey). ${ }^{\star \star} \mathrm{P}<0.002 ;{ }^{\star \star \star} \mathrm{p}<0.0002 ;{ }^{* \star \star \star} \mathrm{p}<0.0001$; ns, no significance. Western blot analysis showing the ubiquitination of endogenous PD-L1 in RKO cells (E) and exogenous PD-L1 in HCT116 ectopic PD-L1 stable cells (F). Western blot analysis evaluating the expression of endogenous PD-L1 (G) and exogenous PD-L1 $(\mathrm{H})$ in the indicated cells transfected with XTP3-B, HRD1, OS-9 and THADA siRNAs as denoted. Co-immunoprecipitation (Co-IP) assays revealing the interactions between XTP3-B/HRD1 and endogenous PD-L1 (I)/exogenous PD-L1 $(\mathrm{J})$ in the indicated cells. The experiments were repeated three times independently with similar results, respectively. (K) Schematic displaying that THADA knockdown blocks PD-L1 trafficking and detains PDL1 in the ER, thereby causing PD-L1 to be recognized, targeted and retrotranslocated by ERAD complex containing E3 ligase HRD1 and lectin-like protein XTP3-B, and ultimately degraded by ubiquitin-proteasome pathway in the cytoplasm. 
A

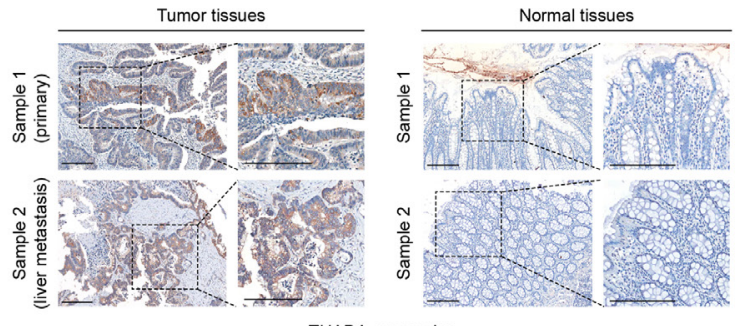

Normal tissues
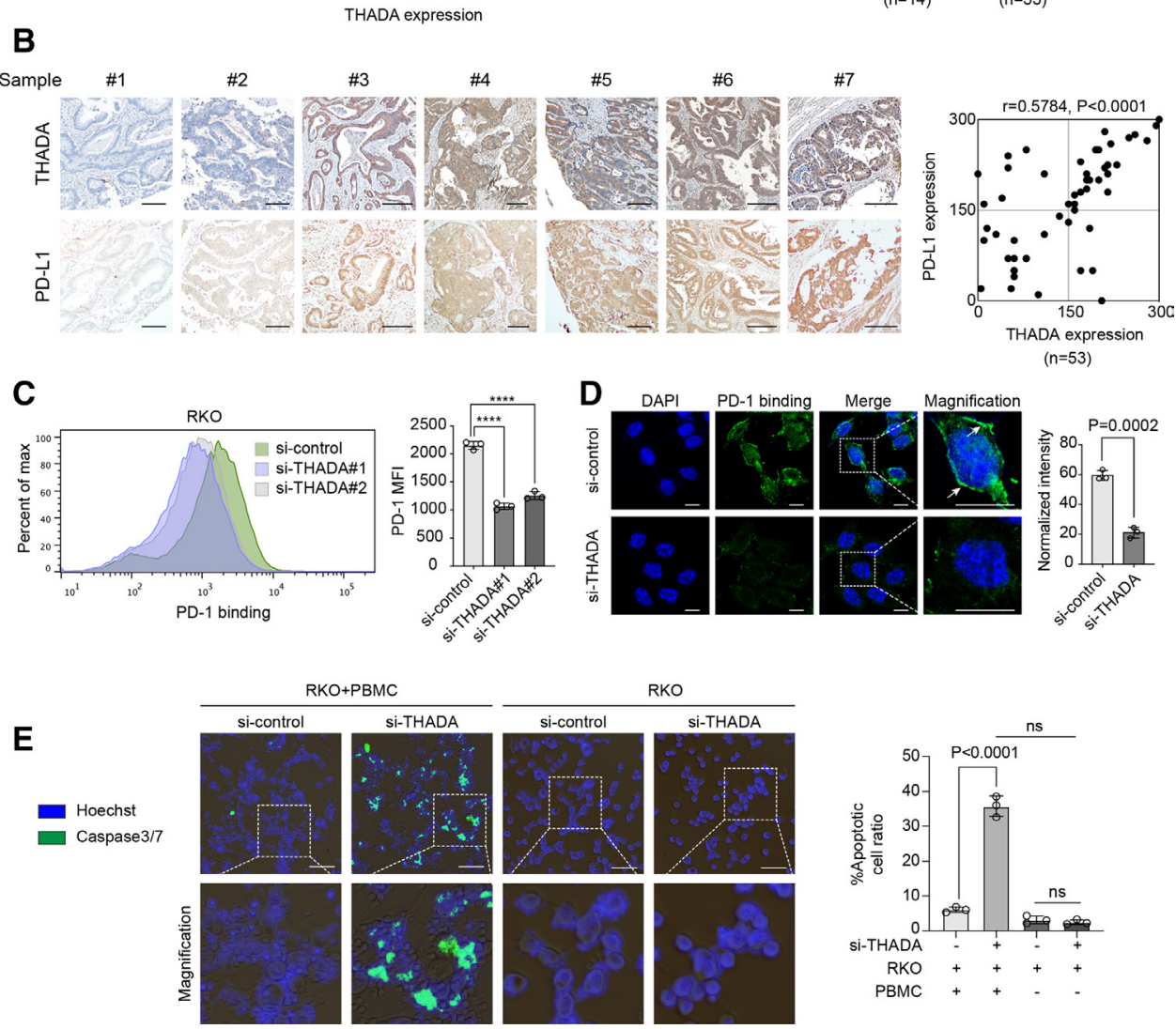

Figure 5 Depletion of thyroid adenoma associated gene (THADA) promoted T cell-mediated antitumor effect. (A) Left, representative immunohistochemistry $(\mathrm{IHC})$ results of colorectal cancer (CRC) tissue microarrays displaying THADA expression in tumor tissues and normal tissues. Scale bars, $200 \mu \mathrm{m}$. Right, statistical result for THADA expression assessed using $\mathrm{H}$ score. The $p$ value was determined by two-sided Student's t-test. (B) Left, representative IHC results of CRC tissue microarrays displaying the expression of THADA and programmed death-ligand 1 (PD-L1) in tumor tissues of the same cases. Scale bars, $200 \mu \mathrm{m}$. Right, statistical result for THADA and PD-L1 expression assessed using H score. The $\mathrm{p}$ value was determined by twosided Pearson's correlation $(p<0.0001)$. (C) Left, flow cytometry examining programmed cell death 1 (PD-1) binding on RKO cells transfected with THADA small interfering RNAs (siRNAs). Right, quantification of mean fluorescence intensity (MFI) of PD1. Values are means $\pm S D$ from three independent experiments. Statistical differences were evaluated by analysis of variance (ANOVA) post hoc test (Tukey). ${ }^{* * * *} \mathrm{P}<0.0001$. (D) Left, immunofluorescence revealing PD-1 binding on RKO cells transfected with THADA siRNAs. White dashed boxes denote the representative fields to be magnified, and the arrows indicate the binding sites. Scale bars, $10 \mu \mathrm{m}$. Right, values are mean $\mathbf{S}$ SD from three independent experiments. Statistical differences were evaluated by two-sided Student's t-test. (E) T cell killing assay. Left, cell nuclei were stained with Hoechst (blue), and apoptotic cells were stained with fluorescent dyes of caspase3/7 cleavage (green). The fluorescence images were merged with phasecontrast images of the cultured RKO cells. White dashed boxes denote the representative fields to be magnified. Scale bars, $50 \mu \mathrm{m}$. Right, values are means $\pm S D$ from $n=3$ independent experiments. The $p$ values were evaluated by ANOVA post hoc test (Tukey); ns, no significance.

tissue microarrays. IHC staining for THADA and PD-L1 expression was assessed using $\mathrm{H}$ score with the formula (weak) $\% \times 1+($ moderate $) \% \times 2+($ strong $) \% \times 3$, ranging from 0 to 300 . As a result, we found THADA expression was increased in tumor tissues compared with normal tissues (figure 5A). Particularly, the expression of THADA was significantly positive correlated with PD-L1 expression in tumor tissues (figure 5B), indicating THADA might be involved in the maintenance of PD-L1 expression in tumor tissues.

Studies have widely demonstrated that PD-L1 expressed on tumor cells facilitates the dysfunction of $\mathrm{T}$ cell-mediated immunosurveillance by PD-1 binding, and blockade of PD-1/PD-L1 pathway efficiently revives 
T cells for tumor killing. ${ }^{16} 172325$ To validate whether THADA-mediated suppression of PD-L1 could functionally promote antitumor immunity, we incubated THADAdepleted tumor cells with recombinant human PD-1 FC fragment to test PD-1 binding activity. As expected, FACS (figure 5C) and immunofluorescence assays (figure 5D) revealed that THADA knockdown caused a reduction in PD-1 binding to tumor cells. T cell killing assays ${ }^{2325}$ were also performed by co-incubating tumor cells with activated PBMCs. As a result, we found that depletion of THADA markedly promoted tumor cell apoptosis, while it exerted no effect in the absence of PBMCs (figure 5E), indicating the pro-apoptotic effects of THADA knockdown on tumor cells is dependent on enhancing $\mathrm{T}$ cell cytotoxity. Taken together, these findings suggest that silencing THADA in PD-L1-abundant tumor cells effectively represses PD-1 binding and provokes $\mathrm{T}$ cell killing, thereby promoting PD-L1-dependent antitumor immunity.

\section{Targeting THADA reinforced PD-L1-dependent tumor immune response in vivo}

Next, we investigated whether THADA depletion enhanced PD-L1-dependent antitumor immune response in vivo. Mouse MC38 CRC cells were infected with THADA shRNA or control shRNA lentivirus. A rescue condition was also established by simultaneously silencing THADA and overexpressing mouse PD-L1. Western blot analyses confirm that THADA knockdown specifically suppressed PD-L1 protein without compromising MHC-I expression, while ectopic expression of PD-L1 could restore PD-L1 reduction caused by THADA absence (figure 6A). RT-PCR revealed that silencing THADA displayed no effect on PD-L1 mRNA expression (figure 6B). CCK8 viability assays were performed to test the impact of THADA knockdown on cultured MC38 tumor cells. Results revealed no appreciable difference in proliferation rate among three groups, implying THADA knockdown did not influence tumor cell proliferation (figure 6C).

Then, the aforementioned MC38 stable clones were, respectively, inoculated subcutaneously into C57BL/6 mice $(n=8)$. The results showed that depletion of THADA strikingly alleviated tumor growth, while the suppression could be rescued in sh-THADA/PD-L1-overexpession group (figure $6 \mathrm{D}-\mathrm{F}$ and online supplemental figure 4A,B). Western blot analysis of tumor tissues confirmed that PD-L1 expression considerably decreased in THADA-knockdown group, while recovered in the rescue group (figure 6G). Similarly, MHC-I expression showed no change. The results were consistent with the in vitro results mentioned above. Analyses of tumor tissues by IHC also showed that PD-L1 expression was downregulated in THADA-knockdown group compared with the control and rescue groups (figure $6 \mathrm{H}$ ). Meanwhile, increased infiltration of CD8+ T cells was detected in THADAknockdown group compared with the other two groups (figure 6I). As CD8+ T cells exerted antitumor effect by secreting granzyme $\mathrm{B}$ to promote tumor cell apoptosis, ${ }^{62}$ we tested CD8+ Tcell infiltration as well as granzyme B release in THADA-knockdown tumor tissues by immunofluorescence. Results showed increased infiltration of CD8+ $\mathrm{T}$ cells accompanied with granzyme $\mathrm{B}$ release in THADA-knockdown group compared with the control and the rescue group (figure 6J).

To assess the effect of THADA knockdown, mice inoculated MC38 stable clones (THADA shRNA or control shRNA lentivirus) were randomized to receive anti-PD-L1 antibody or isotype-matched control antibody $(\mathrm{n}=8$ per group). As a result, we found that silencing THADA and anti-PD-L1 antibody both displayed significant therapeutic effects, and targeting THADA alone was sufficient to completely suppress tumor growth (figure $6 \mathrm{~K}-\mathrm{M}$ and online supplemental figure 4C,D). Particularly, we test the synergistic effect of the combination treatment of shTHADA and anti-PD-L1 drugs, and the results revealed enhancement in antitumor effect. Although shTHADA alone displayed strong effect, the combination treatment further decreased tumor sizes. Analyses of tumor tissues by IHC showed PD-L1 expression was downregulated in THADA-knockdown group and THADAknockdown+anti-PD-L1 treatment group. Interestingly, we found that monotherapy with anti-PD-L1 drug did not affect PD-L1 expression (online supplemental figure $4 \mathrm{E})$. Meanwhile, increased infiltration of CD8+ T cells and release of granzyme B was detected in anti-PD-L1 treatment group, THADA-knockdown group as well as the combination treatment group (online supplemental figure $4 \mathrm{~F}, \mathrm{G})$. Collectively, these findings reveal that disruption of THADA remarkably attenuates tumor growth by suppressing PD-L1 and promoting CD8 T cell activity in MC38 tumor models, highlighting THADA as a promising target to reinforce the immunity response of PD-L1-dependent tumors.

\section{DISCUSSION}

In this study, we identified THADA as a key regulator of PD-L1 maturation. THADA depletion significantly caused PD-L1 degradation, thereby functionally blocking PD-1 binding and reviving $\mathrm{T}$ cell cytotoxicity as well as repressing tumor growth in mouse MC38 tumor models, suggesting THADA as an alternative target for optimizing PD-L1-dependent tumor immunotherapy.

The molecular mechanisms of PD-L1 have been extensively studied, involving genomic and epigenetic alterations, transcriptional and post-transcriptional modifications as well as exosomal regulation. ${ }^{2} 1415236364$ As a type I transmembrane protein, PD-L1 undergoes exquisite secretory pathway throughout the lifetime. Abundant studies have demonstrated multiple PD-L1 trafficking mechanisms from the ER processing ${ }^{16-22}$ to the dynamic endosomal trafficking. ${ }^{12} 1415$ 23-26 $\mathrm{A}$ substantial number of PD-L1 proteins get modified in the ER, however, how PD-L1 is exported to the Golgi is rarely reported yet. Here, we shed new light on the ER-to-Golgi transport mechanism of PD-L1. THADA serves as a specific intermediator to couple PD-L1 to Sec24A, the core module of COPII 
A

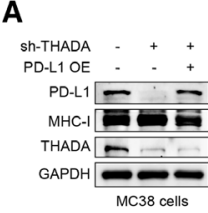

DC38 tumor growth

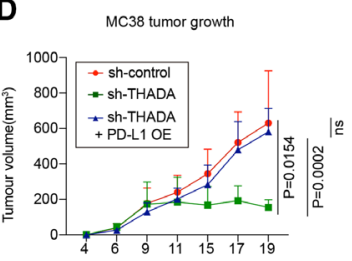

Days after injection

G

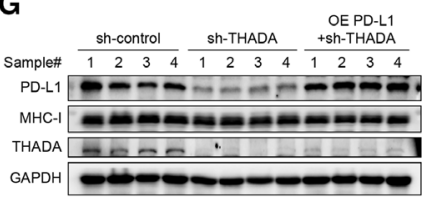

B mTHADAMRNA

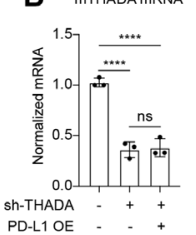

E

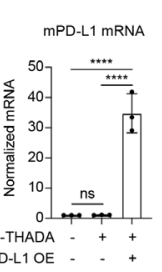

C

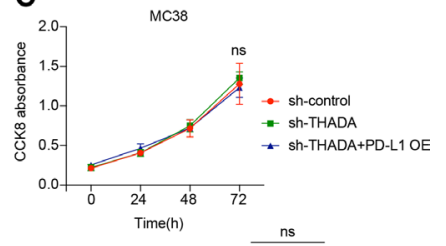

F $\quad{ }_{0.8} \quad \frac{\mathrm{ns}}{\mathrm{P}=0.0416}$

Resected tumors
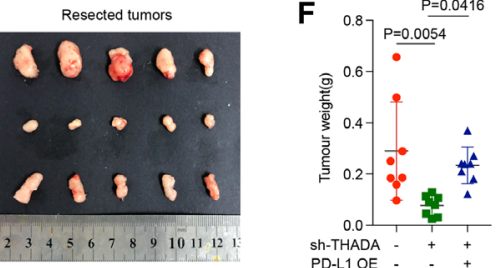

H

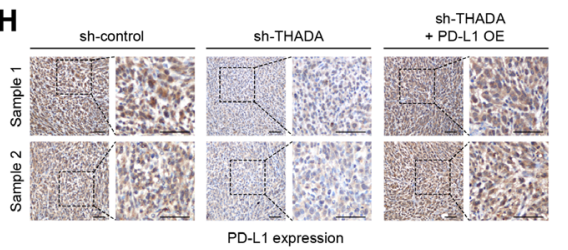

PD-L1 expression

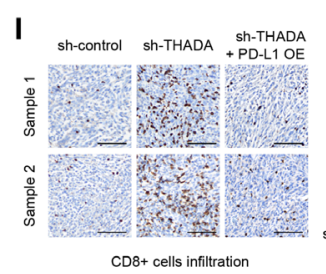

I

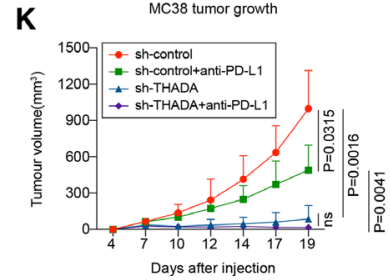

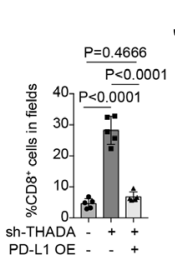
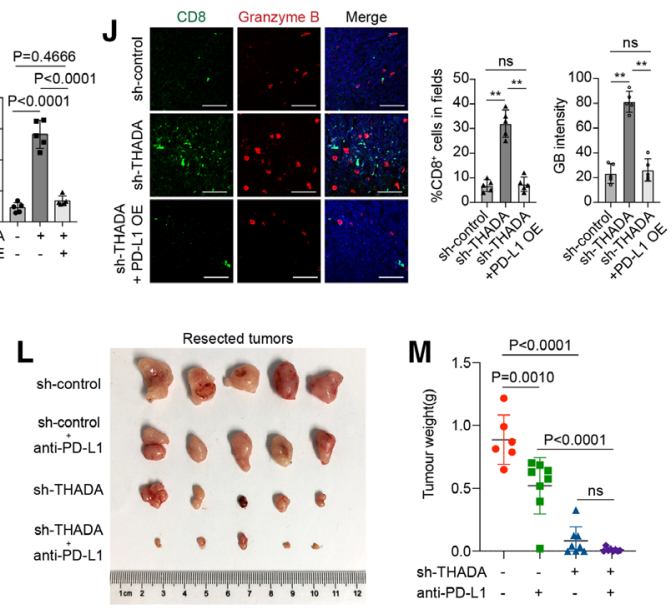

Figure 6 Targeting thyroid adenoma associated gene (THADA) improved programmed death-ligand 1 (PD-L1)-dependent tumor immune response in vivo. (A) Western blot analysis testing the expression of PD-L1, major histocompatibility complex (MHC)-I and THADA in indicated MC38 stable clones used for establishing tumor models. The experiment was repeated three times independently with similar results. (B) RT-PCR showing the mRNA expression of mouse THADA (left) and mouse PDL1 (right) in indicated MC38 stable clones. Values are means $\pm S D$ from $n=3$ independent experiments. Statistical differences were evaluated by analysis of variance (ANOVA) post hoc test (Tukey). ${ }^{* \star \star *} \mathrm{P}<0.0001$; ns, no significance. (C) CCK8 viability assay examining the proliferation rate of indicated cultured MC38 stable clones. The experiment was repeated three times independently with similar results. Statistical differences were determined by ANOVA test. (D) Tumor growth rate of each group inoculated with indicated MC38 stable clones. The dots denote means and 95\% Cls (two-way ANOVA; $n=8$ per group). (E) The images of resected tumors from mice. (F) Tumor weight of each individual. Values are means $\pm S D$ ( $n=8$ per group). Statistical differences were evaluated by ANOVA post hoc test (Tukey). (G) Western blot analysis showing the expression of PD-L1, MHC-I and THADA in indicated tumor tissues. (H) Immunohistochemistry assay showing mouse PD-L1 expression in indicated tumor tissues. Dashed boxes denote the representative fields to be magnified. Scale bars, $50 \mu \mathrm{m}$. (I) Left, immunohistochemistry assay revealing CD8+ cell infiltration in indicated tumor tissues. Scale bars, $100 \mu \mathrm{m}$. Right, quantification of CD8+ cell infiltration in indicated tumor tissues. Values are means $\pm S D$ from five independent samples from each group. The $p$ value was determined by ANOVA post hoc test. (J) Immunofluorescence assays showing CD8+ Tcell infiltration and granzyme B release in indicated tumor tissues. Scale bars, $50 \mu \mathrm{m}$. Values are means $\pm S D$ from five independent samples of each group. Statistical differences were evaluated by ANOVA post hoc test (Tukey). ${ }^{\star \star} \mathrm{P}<0.0001$; ns, no significance. (K) Tumor growth rate of each group that received indicated treatment after inoculation of indicated MC38 stable clones. The points indicate means and 95\% Cls (twoway ANOVA; $n=8$ per group). (L) Images of resected tumors from mice. (M) Tumor weight. Values are means $\pm S D$ and statistical differences were determined by ANOVA post hoc test (Tukey).

machinery, thereby driving PD-L1 export to the Golgi and maintaining the expression of PD-L1 in tumor cells. The absence of THADA strikingly blocks the interaction between PD-L1 and Sec24A, causing ER retention of PD-L1 and thus exhaustion of Golgi and cytomembrane resident PD-L1. Aberrant retention of PD-L1 proteins 


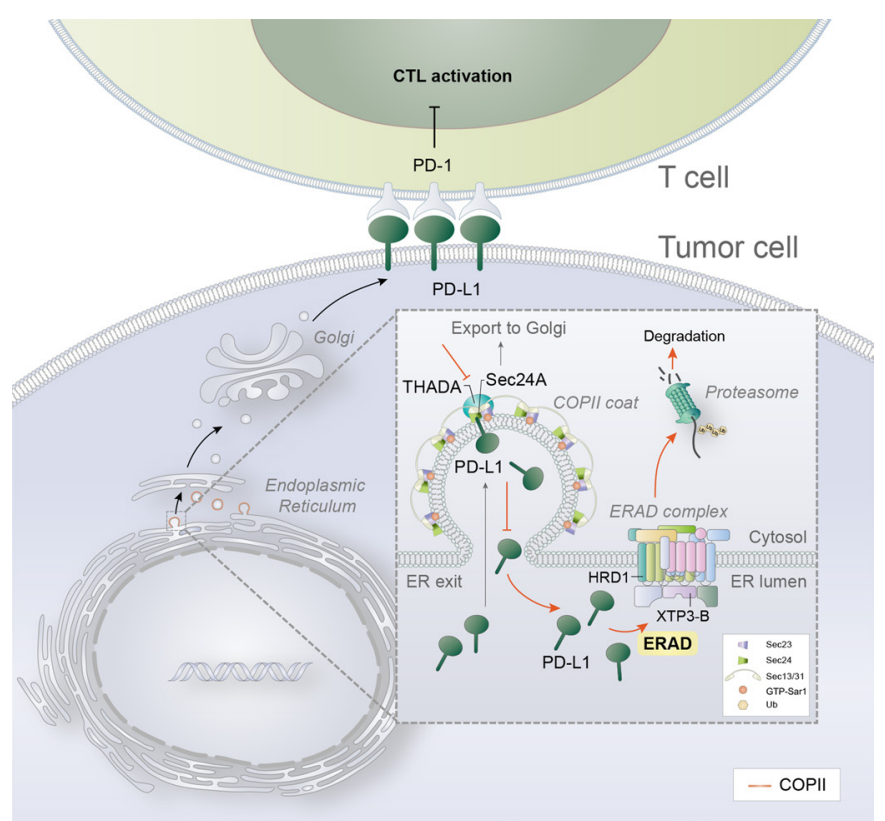

Figure 7 Targeting thyroid adenoma associated gene (THADA) blocks programmed death-ligand 1 (PD-L1) trafficking to Golgi while induces endoplasmic reticulum (ER) retention of PD-L1 and ER-associated degradation (ERAD). THADA mediates the interaction of PD-L1 and Sec24A, driving PD-L1 export from the ER by coat protein complex II (COPII) trafficking vesicles. THADA depletion significantly blocks PD-L1 binding to Sec24A, thereby causing ER retention of PD-L1 and exhaustion of both Golgi and cytomembrane resident PD-L1. Aberrant aggregation of PD-L1 proteins trigger ERAD, which are recognized and retrotranslocated by HRD1 ERAD complex, and ultimately eliminated by ubiquitin-proteasome pathway in the cytoplasm.

in the ER lumen are subsequently recognized, targeted and retrotranslocated by HRD1 ERAD complex via ERAD pathway, and ultimately eliminated by the ubiquitinproteasome system in the cytoplasm (figure 7).

As one of the five key subunits of COPII coat machinery, Sec24 exclusively determines the selectivity of the cargo protein for COPII vesicles. There are four isoforms of human Sec24 family, whereas whether there is functional difference and redundancy among the four isoforms remains unclear. ${ }^{30-34}$ In this work, we identified Sec24 homologue A was responsible for binding PD-L1 to COPII vesicles. Particularly, we found that such specific targeting of PD-L1 by Sec24A required a novel cargo adaptor THADA, while depletion of THADA suppressed the interaction between PD-L1 and Sec24A. Such selective cargo adaptor markedly enhances the sorting efficiency and accuracy of COPII vesicle transportation, shedding new light on the sophisticated PD-L1 trafficking machineries.

ER-resident lectin-like proteins OS-9 and XTP3-B are both proposed to participate in the recognition and retrotranslocation of ERAD substrates, although XTP3-B is less well documented and proposed to be functionally redundant or even against ERAD. ${ }^{58-60}$ A previous study showed OS-9, XTP3-B could both regulate the ERAD of aberrant
glycosylated-PD-L1. ${ }^{18}$ In the present study, we found that depleting XTP3-B in THADA knockdown RKO cells could remarkably restore the expression of PD-L1, while OS-9 depletion did not have the same effect (figure 4G). Additionally, we noticed that XTP3-B depletion increased OS-9 expression regardless of the absence or presence of THADA, while silencing OS-9 seemed to have less effect on XTP3-B expression (figure 4G and online supplemental figure 3D). Our results revealed XTP3-B rather than OS-9 modulated the ERAD degradation of PD-L1 when THADA was knockdown. There seems to be a compensatory regulation between OS-9 and XTP3-B, but the underlying mechanism needs further investigation. In addition, in consistent with the previous study, ${ }^{18}$ we also identified ERAD E3 ligase HRD1 could physically interact with PD-L1 and mediate its ERAD degradation.

THADA is mainly reported to participate in the regulation of organismal metabolism and associated with diabetes and polycystic ovary syndrome. ${ }^{65-68}$ Although several studies correlated THADA with an increased risk of multiple malignancies, the function and molecular mechanism of THADA in tumors is still ill-defined. ${ }^{49-52}$ Our work identifies THADA as a key regulator of PD-L1 maintenance, and THADA expression in CRC tissues is positive correlated with PD-L1 expression. Silencing THADA specifically suppresses PD-L1 and promotes antitumor effect by enhancing $\mathrm{T}$ cell cytotoxicity, while has no impact on MHC-I expression or cell proliferation. Thus, we consider THADA as a safe and ideal target to selectively suppress PD-L1 and minimally compromise the function of normal tissues.

Although existing antibody inhibitors for PD-L1 can efficiently block the PD-L1 functions on cell surface, recent studies have found that acquired resistance may be caused by endocytosis of the antibody-receptor complex and repopulation of intracellular PD-L1 onto the cell surface. Rather than inhibiting PD-L1 on the cell surface, a more attractive strategy is to deplete PD-L1 within the whole tumor cell. We found that THADA is critically required for maintaining PD-L1 expression, and targeting THADA induced efficient PD-L1 degradation and sustained functional inhibition. Our proof-of-concept study may inspire future researches aimed to develop new PD-L1 inhibitors. It is to note that continuous efforts by both biologists and medical chemists may be required to eventually develop a clinically effective and safe PD-L1 degrader, in order to reinforce or complement the effects of anti-PD-L1 therapy.

\section{CONCLUSIONS}

In summary, our study unveils a novel transport mechanism for PD-L1 maturation and a critical regulator modulating ER-to-Golgi trafficking of PD-L1 across diverse cancer types. THADA specifically regulates the interaction of PD-L1 and Sec24A-dependent COPII vesicles, without compromising MHC-I expression. Blockade of THADA significantly degraded PD-L1 via ERAD pathway. 
The work highlights THADA and the associated pathway as a promising therapeutic target to improve the efficacy of anti-PD-L1 therapy.

\section{Author affiliations}

${ }^{1}$ Zhongshan-Xuhui Hospital, Institutes of Biomedical Sciences (visiting), Shanghai Key Laboratory of Medical Epigenetics, International Co-laboratory of Medical Epigenetics and Metabolism (Ministry of Science and Technology), Fudan University, Shanghai, China

${ }^{2}$ Division of Gastroenterology and Hepatology, Renji Hospital, School of Medicine, Shanghai Jiao Tong University, Shanghai, China

${ }^{3}$ Zhongshan-Xuhui Hospital, Shanghai Xuhui Central Hospital, Institutes of Biomedical Sciences, Shanghai Key Laboratory of Medical Epigenetics, International Co-laboratory of Medical Epigenetics and Metabolism (Ministry of Science and Technology), Fudan University, Shanghai, China

${ }^{4}$ Translational Research Institute of Brain and Brain-Like Intelligence, Shanghai Fourth People's Hospital, School of Medicine, Tongji University, Shanghai 200434, China

${ }^{5}$ Innomodels Biotechnology (Beijing) Co., Ltd, Beijing, China

Correction notice This paper has been updated since first published to add author details for author Ke Xu.

Contributors CL, HC, SD, KX, HW, HY, YW, DC, XG and FH performed/arranged experiments and analyzed data. J-YF provided supports on study resources. $C L$ and JX wrote the paper. JX conceived the study.

Funding This work was supported by National Key R \& D Program of China (2016YFC0906002), National Natural Science Foundation of China (No: 82030104, $81874050,81572326)$, Basic Research Projects of Shanghai Science and Technology Innovation Action Plan (20JC1410700); Tang Scholar (JX), and Startup Research Funding of Fudan University. This work was also partially supported by Shanghai Sailing Program (20YF1453100), Shanghai Health Commission Clinical Research Special Youth Project (20204Y0318), Fundamental Research Funds for the Central Universities (Tongji University).

Competing interests None declared.

Patient consent for publication Not required.

Ethics approval All animal experiments were performed in strict accordance with the relevant ethical guidelines, approved by the Department of Laboratory Animal Science of Fudan University and the Institutional Animal Care and Use Committee of Renji Hospital, School of Medicine, Shanghai Jiaotong University. CRC tissue microarray was purchased from BioChip (Shanghai, China) with informed patient consent provided. The tissue microarray (XT13-050) involved matched normal tissues as well as primary and metastatic tumor tissue samples from 22 patients (more than one metastatic tumor in some of the patients). The study was approved by the Ethics Committee of Fudan University.

Provenance and peer review Not commissioned; externally peer reviewed.

Data availability statement Data are available on reasonable request. All data relevant to the study are included in the article or uploaded as supplementary information. The authors declare that all data supporting the findings of this study are available within the paper and its supplementary information files.

Supplemental material This content has been supplied by the author(s). It has not been vetted by BMJ Publishing Group Limited (BMJ) and may not have been peer-reviewed. Any opinions or recommendations discussed are solely those of the author(s) and are not endorsed by BMJ. BMJ disclaims all liability and responsibility arising from any reliance placed on the content. Where the content includes any translated material, BMJ does not warrant the accuracy and reliability of the translations (including but not limited to local regulations, clinical guidelines, terminology, drug names and drug dosages), and is not responsible for any error and/or omissions arising from translation and adaptation or otherwise.

Open access This is an open access article distributed in accordance with the Creative Commons Attribution Non Commercial (CC BY-NC 4.0) license, which permits others to distribute, remix, adapt, build upon this work non-commercially, and license their derivative works on different terms, provided the original work is properly cited, appropriate credit is given, any changes made indicated, and the use is non-commercial. See http://creativecommons.org/licenses/by-nc/4.0/.

\section{ORCID iD}

Jie Xu http://orcid.org/0000-0001-9163-3898
REFERENCES

$1 \mathrm{Xu}$ J. Regulation of cancer immune checkpoints. Singapore: Springer-Verlag, 2020.

2 Sun C, Mezzadra R, Schumacher TN. Regulation and function of the PD-L1 checkpoint. Immunity 2018;48:434-52.

3 Kaplon H, Reichert JM. Antibodies to watch in 2019. MAbs 2019;11:219-38.

4 Gong J, Chehrazi-Raffle A, Reddi S, et al. Development of PD-1 and PD-L1 inhibitors as a form of cancer immunotherapy: a comprehensive review of registration trials and future considerations. $\mathrm{J}$ Immunother Cancer 2018;6:8.

5 Kon E, Benhar I. Immune checkpoint inhibitor combinations: current efforts and important aspects for success. Drug Resist Updat 2019;45:13-29.

6 Syn NL, Teng MWL, Mok TSK, et al. De-novo and acquired resistance to immune checkpoint targeting. Lancet Oncol 2017;18:e731-41.

7 Zhuang Y, Liu C, Liu J, et al. Resistance mechanism of PD-1/ PD-L1 blockade in the Cancer-Immunity cycle. Onco Targets Ther 2020;13:83-94.

8 Baxi S, Yang A, Gennarelli RL, et al. Immune-related adverse events for anti-PD-1 and anti-PD-L1 drugs: systematic review and metaanalysis. BMJ 2018;360:k793.

9 Sharma P, Hu-Lieskovan S, Wargo JA, et al. Primary, adaptive, and acquired resistance to cancer immunotherapy. Cell 2017;168:707-23.

10 Page DB, Postow MA, Callahan MK, et al. Immune modulation in cancer with antibodies. Annu Rev Med 2014;65:185-202.

11 Hui E, Cheung J, Zhu J, et al. T cell costimulatory receptor CD28 is a primary target for PD-1-mediated inhibition. Science 2017;355:1428-33.

12 Burr ML, Sparbier CE, Chan Y-C, et al. CMTM6 maintains the expression of PD-L1 and regulates anti-tumour immunity. Nature 2017;549:101-5

13 Tu X, Qin B, Zhang Y, et al. PD-L1 (B7-H1) competes with the RNA exosome to regulate the DNA damage response and can be targeted to sensitize to radiation or chemotherapy. Mol Cell 2019;74:1215-26.

14 Chen G, Huang AC, Zhang W, et al. Exosomal PD-L1 contributes to immunosuppression and is associated with anti-PD-1 response. Nature 2018;560:382-6.

15 Poggio M, Hu T, Pai C-C, et al. Suppression of exosomal PDL1 induces systemic anti-tumor immunity and memory. Cell 2019;177:414-27.

16 Li C-W, Lim S-O, Xia W, et al. Glycosylation and stabilization of programmed death ligand-1 suppresses T-cell activity. Nat Commun 2016;7:12632.

17 Li C-W, Lim S-O, Chung EM, et al. Eradication of triple-negative breast cancer cells by targeting glycosylated PD-L1. Cancer Cell 2018;33:187-201.

18 Cha J-H, Yang W-H, Xia W, et al. Metformin promotes antitumor immunity via endoplasmic-reticulum-associated degradation of PDL1. Mol Cell 2018:71:606-20.

19 Verdura S, Cuyàs $\mathrm{E}$, Cortada $\mathrm{E}$, et al. Resveratrol targets PD-L1 glycosylation and dimerization to enhance antitumor T-cell immunity. Aging 2020;12:8-34.

20 Chen F-F, Li Z, Ma D, et al. Small-molecule PD-L1 inhibitor BMS1166 abrogates the function of PD-L1 by blocking its ER export. Oncoimmunology 2020;9:1831153.

21 Chou C-W, Yang R-Y, Chan L-C, et al. The stabilization of PD-L1 by the endoplasmic reticulum stress protein GRP78 in triple-negative breast cancer. Am J Cancer Res 2020;10:2621-34.

22 Maher CM, Thomas JD, Haas DA, et al. Small-Molecule sigma1 modulator induces autophagic degradation of PD-L1. Mol Cancer Res 2018;16:243-55.

23 Yao H, Lan J, Li C, et al. Inhibiting PD-L1 palmitoylation enhances T-cell immune responses against tumours. Nat Biomed Eng 2019;3:306-17.

$24 \mathrm{Li} \mathrm{C}$, Yao $\mathrm{H}$, Wang $\mathrm{H}$, et al. Repurposing screen identifies amlodipine as an inducer of PD-L1 degradation and antitumor immunity. Oncogene 2021;40:1128-46.

25 Wang $\mathrm{H}$, Yao H, Li C, et al. HIP1R targets PD-L1 to lysosomal degradation to alter T cell-mediated cytotoxicity. Nat Chem Biol 2019;15:42-50.

26 Monypenny J, Milewicz H, Flores-Borja F, et al. Alix regulates tumormediated immunosuppression by controlling EGFR activity and PD-L1 presentation. Cell Rep 2018;24:630-41.

27 Lord C, Ferro-Novick S, Miller EA. The highly conserved COPII coat complex sorts cargo from the endoplasmic reticulum and targets it to the Golgi. Cold Spring Harb Perspect Biol 2013;5. doi:10.1101/ cshperspect.a013367. [Epub ahead of print: 01 Feb 2013].

28 Béthune J, Wieland FT. Assembly of COPI and COPII vesicular coat proteins on membranes. Annu Rev Biophys 2018;47:63-83. 
29 Russell C, Stagg SM. New insights into the structural mechanisms of the COPII coat. Traffic 2010;11:303-10.

30 Miller EA, Beilharz TH, Malkus PN, et al. Multiple cargo binding sites on the COPII subunit Sec24p ensure capture of diverse membrane proteins into transport vesicles. Cell 2003;114:497-509.

31 Mossessova E, Bickford LC, Goldberg J. SNARE selectivity of the COPII coat. Cell 2003:114:483-95.

32 Wendeler MW, Paccaud J-P, Hauri H-P. Role of SEC24 isoforms in selective export of membrane proteins from the endoplasmic reticulum. EMBO Rep 2007;8:258-64.

33 Mancias JD, Goldberg J. Structural basis of cargo membrane protein discrimination by the human COPII coat machinery. Embo $J$ 2008;27:2918-28

34 Mancias JD, Goldberg J. The transport signal on Sec22 for packaging into COPII-coated vesicles is a conformational epitope. Mol Cell 2007;26:403-14.

35 Powers J, Barlowe C. Erv14p directs a transmembrane secretory protein into COPIl-coated transport vesicles. Mol Biol Cell 2002;13:880-91.

36 Herzig Y, Sharpe HJ, Elbaz Y, et al. A systematic approach to pair secretory cargo receptors with their cargo suggests a mechanism for cargo selection by Erv14. PLoS Biol 2012;10:e1001329.

37 Pagant S, Wu A, Edwards S, et al. Sec24 is a coincidence detector that simultaneously binds two signals to drive ER export. Curr Biol 2015;25:403-12.

38 Castro CP, Piscopo D, Nakagawa T, et al. Cornichon regulates transport and secretion of TGFalpha-related proteins in metazoan cells. J Cell Sci 2007;120:2454-66.

39 Schwenk J, Harmel N, Zolles G, et al. Functional proteomics identify cornichon proteins as auxiliary subunits of AMPA receptors. Science 2009;323:1313-9.

40 Sauvageau E, Rochdi MD, Oueslati M, et al. CNIH4 interacts with newly synthesized GPCR and controls their export from the endoplasmic reticulum. Traffic 2014:15:383-400.

41 Goldstein JL, DeBose-Boyd RA, Brown MS. Protein sensors for membrane sterols. Cell 2006;124:35-46.

42 Zhang Y, Motamed M, Seemann J, et al. Point mutation in luminal loop 7 of Scap protein blocks interaction with loop 1 and abolishes movement to Golgi. J Biol Chem 2013;288:14059-67.

$43 \mathrm{Xu} \mathrm{C,} \mathrm{Ng} \mathrm{DTW.} \mathrm{Glycosylation-directed} \mathrm{quality} \mathrm{control} \mathrm{of} \mathrm{protein}$ folding. Nat Rev Mol Cell Biol 2015;16:742-52

44 Ferris SP, Kodali VK, Kaufman RJ. Glycoprotein folding and qualitycontrol mechanisms in protein-folding diseases. Dis Model Mech 2014;7:331-41.

45 Vembar SS, Brodsky JL. One step at a time: endoplasmic reticulum-associated degradation. Nat Rev Mol Cell Biol 2008;9:944-57.

46 Dorand RD, Nthale J, Myers JT, et al. Cdk5 disruption attenuates tumor PD-L1 expression and promotes antitumor immunity. Science 2016;353:399-403.

47 Wang XF, Lei Y, Chen M, et al. PD-1/PDL1 and CD28/CD80 pathways modulate natural killer $\mathrm{T}$ cell function to inhibit hepatitis $\mathrm{B}$ virus replication. J Viral Hepat 2013;20 Suppl 1:27-39.

48 Deng R, Cassady K, Li X, et al. B7H1/CD80 interaction augments PD-1-dependent $T$ cell apoptosis and ameliorates graft-versus-host disease. J Immunol 2015;194:560-74.
49 Cheng I, Caberto CP, Lum-Jones A, et al. Type 2 diabetes risk variants and colorectal cancer risk: the Multiethnic cohort and page studies. Gut 2011;60:1703-11.

50 Zhao F, Xu Y, Yang K, et al. THADA gene polymorphism and prostate cancer risk: a meta-analysis. Oncol Res Treat 2014;37:106-10.

$51 \mathrm{Li}$ X-H, Xu Y, Yang K, et al. Association of THADA, FOXP4, GPRC6A RFX6 genes and 8q24 risk alleles with prostate cancer in northern Chinese men. J Buon 2015;20:1223-8.

52 Panebianco F, Kelly LM, Liu P, et al. THADA fusion is a mechanism of IGF2BP3 activation and IGF1R signaling in thyroid cancer. Proc Natl Acad Sci U S A 2017;114:2307-12.

53 Garcia-Diaz A, Shin DS, Moreno BH, et al. Interferon receptor signaling pathways regulating PD-L1 and PD-L2 expression. Cell Rep 2017;19:1189-201.

54 Huttlin EL, Ting L, Bruckner RJ, et al. The BioPlex network: a systematic exploration of the human interactome. Cell 2015;162:425-40.

55 Huttlin EL, Bruckner RJ, Paulo JA, et al. Architecture of the human interactome defines protein communities and disease networks. Nature 2017;545:505-9.

56 Havugimana PC, Hart GT, Nepusz T, et al. A census of human soluble protein complexes. Cell 2012;150:1068-81.

57 Barlowe C, Helenius A. Cargo capture and bulk flow in the early secretory pathway. Annu Rev Cell Dev Biol 2016;32:197-222.

58 Bernasconi R, Galli C, Calanca V, et al. Stringent requirement for HRD1, SEL1L, and OS-9/XTP3-B for disposal of ERAD-LS substrates. J Cell Biol 2010;188:223-35.

59 Christianson JC, Shaler TA, Tyler RE, et al. OS-9 and GRP94 deliver mutant alpha1-antitrypsin to the Hrd1-SEL1L ubiquitin ligase complex for ERAD. Nat Cell Biol 2008;10:272-82.

60 Fujimori T, Kamiya Y, Nagata K, et al. Endoplasmic reticulum lectin XTP3-B inhibits endoplasmic reticulum-associated degradation of a misfolded $\alpha 1$-antitrypsin variant. Febs J 2013;280:1563-75.

61 Wang HB, Yao H, Li CS, et al. Rise of PD-L1 expression during metastasis of colorectal cancer: implications for immunotherapy. $J$ Dig Dis 2017;18:574-81.

62 Chowdhury D, Lieberman J. Death by a thousand cuts: granzyme pathways of programmed cell death. Annu Rev Immunol 2008;26:389-420.

63 Wang Y, Wang H, Yao H, et al. Regulation of PD-L1: emerging routes for targeting tumor immune evasion. Front Pharmacol 2018;9:536.

64 Hsu J-M, Li C-W, Lai Y-J, et al. Posttranslational modifications of PD-L1 and their applications in cancer therapy. Cancer Res 2018;78:6349-53.

65 Moraru A, Cakan-Akdogan G, Strassburger K, et al. THADA regulates the organismal balance between energy storage and heat production. Dev Cell 2017;41:72-81.

66 Prasad RB, Lessmark A, Almgren P, et al. Excess maternal transmission of variants in the THADA gene to offspring with type 2 diabetes. Diabetologia 2016;59:1702-13.

67 Goodarzi MO, Jones MR, Li X, et al. Replication of association of DENND1A and THADA variants with polycystic ovary syndrome in European cohorts. J Med Genet 2012:49:90-5.

68 Bakhashab S, Ahmed N. Genotype based risk predictors for polycystic ovary syndrome in Western Saudi Arabia. Bioinformation 2019;15:812-9. 


\section{Correction: THADA drives Golgi residency and upregulation of PD-L1 in cancer cells and provides promising target for immunotherapy}

Li C, Chi H, Deng S, et al. THADA drives Golgi residency and upregulation of PD-L1 in cancer cells and provides promising target for immunotherapy. J Immunother Cancer 2021;9:e002443. doi: 10.1136/jitc-2021-002443

This paper has been updated since first published to add author details for author Ke $\mathrm{Xu}$.

Open access This is an open access article distributed in accordance with the Creative Commons Attribution Non Commercial (CC BY-NC 4.0) license, which permits others to distribute, remix, adapt, build upon this work non-commercially, and license their derivative works on different terms, provided the original work is properly cited, appropriate credit is given, any changes made indicated, and the use is non-commercial. See http://creativecommons.org/licenses/by-nc/4.0/.

C Author(s) (or their employer(s)) 2021. Re-use permitted under CC BY-NC. No commercial re-use. See rights and permissions. Published by BMJ.

J Immunother Cancer 2021;9:e002443orr1. doi:10.1136/jitc-2021-002443orr1

A) Check for updates 